\title{
ANTIUTILITARISMO DI DANTE
}

\author{
Dante's Antiutilitarianism
}

\author{
Marco Romanelli \\ Università dell'Età Libera, Firenze
}

\section{Sommario}

È opinione oggi largamente diffusa che la visione storica e politica di Dante abbia caratteri fortemente conservatori o addirittura reazionari. In sintesi, la polemica dantesca contro l'avarizia e la cupidigia nasconderebbe un radicale rifiuto della modernità e della nuova realtà socioeconomica che si stavano affermando ai suoi tempi, in nome di un ritorno al «buon tempo antico» e al mondo immobile e chiuso della tradizione. In questo saggio intendo dimostrare, al contrario, che l'ipotesi elaborata da Dante è tutt'altro che conservatrice, ma propone invece un modello rivoluzionario di società fondata sui valori del dono e della caritas opposti all'utilitarismo che fino da allora si stava imponendo come principio guida della civiltà occidentale.

\section{Parole chiave}

Avarizia; cortesia; dono; mercante; utilitarismo

\begin{abstract}
It is a widespread opinion that the historical and political vision of Dante has highly conservative or even reactionary characteristics. In brief, Dante's polemics against avarice and cupidity hides a radical refusal of modernity and the new socio-economic reality that was taking place in his time, in the name of a return to "good old days» and to the immobile and closed world of tradition. This article demonstrates that, on the contrary, the hypothesis elaborated by Dante was everything but conservative. He proposed, instead, a revolutionary society founded on the value of the present and of caritas, as opposed to utilitarianism that had been imposed, since then, as a guideline of Western civilization.
\end{abstract}

\section{Keywords}

Avarice; courtesy; present; merchant; utilitarianism 
Nella storia del pensiero cristiano, e segnatamente in quella del cristianesimo medievale, l'esaltazione della povertà è un tema così diffuso ${ }^{1}$ che non può far meraviglia il trovarlo largamente presente nell'opera di Dante. Casomai può lasciare qualche perplessità il fatto che questo tema, correlato con la polemica contro la cupidigia, la logica del profitto, il culto del denaro, il prestito a interesse e l'usura, coniugato insomma con la critica della società mercantile così come si era venuta configurando nel corso del Duecento, costituisca uno degli argomenti più utilizzati da quella radicata opinione che vede in Dante un reazionario ottusamente avverso al mondo nuovo che si era ormai «gloriosamente» imposto nell'Europa del suo tempo (le virgolette esprimono ovviamente il punto di vista di chi scrive), quasi che la parola d'ordine di François Guizot, enrichez-vous!, si fosse propagata con sei secoli di anticipo in un'area che andava dal Mediterraneo al Baltico.

Per la verità, questa rappresentazione di un Dante laudator temporis acti deciso a rifiutare ogni compromesso con la modernità e cristallizzato in una sua allucinata utopia priva di ogni riscontro e di ogni concretezza storica è un'acquisizione critica relativamente recente, le cui prime tracce sono ravvisabili a partire dal secondo dopoguerra e che forse si spiega come una reazione al mito del «poeta nazionale» che dominò dall'Ottocento alla seconda metà del Novecento, attraversando prima l'Italia risorgimentale, poi quella liberale e infine quella fascista, con variazioni significative (dal dantismo patriottico del Risorgimento al dantismo nazionalista postunitario fino a quello imperialista del ventennio) ma comunque sempre ruotanti intorno all'asse di Dante «poeta della nazione». ${ }^{2}$

E del resto, la prova più incontestabile del ruolo nazionale, di guida e di profeta dei destini della patria assegnato unanimemente a Dante si era già avuta nel 1865 con la celebrazione del sesto centenario della nascita del poeta, solennemente tenuta a Firenze da poco divenuta capitale del Regno d'Italia. Come scrive Carlo Dionisotti, «nulla di simile a quella celebrazione si era mai visto prima in Italia, né si vide poi»:3 due anni di preparativi, due settimanali dedicati che uscirono fra il 1864 e il 1865 (il Giornale del centenario di Dante Alighieri e La festa di Dante: letture domenicali del popolo

\footnotetext{
${ }^{1}$ Un'ottima sintesi si trova in Capitani, O. (ed.), La concezione della povertà nel Medioevo, Bologna, Pàtron, 1981. Molto utile per la ricchissima documentazione anche Evangelisti, P., Il pensiero economico nel Medioevo. Ricchezza, povertà, mercato e moneta, Roma, Carocci, 2017.

${ }^{2}$ Per quanto riguarda il Dante «nazionalista», si veda per esempio quanto scrive il Parodi: «Dante non è soltanto il poeta dell'Italia, ma è il suo simbolo più alto; anzi, se come poeta egli appartiene al mondo intiero non meno che a lei, come simbolo appartiene soltanto a lei, e l'Italia deve gelosamente vigilare perché non soffra menomazioni, poiché i grandi come lui sono quasi bandiere, intorno alle quali, nei momenti più solenni o più tragici della storia, una nazione può raccogliersi e sentirsi una di cuore e di volontà», Parodi, E.G., «Benedetto Croce. La metodologia della critica letteraria e la Divina Commedia», Bullettino della Società Dantesca, 2 (1920), p. 2. Quanto al Dante «fascista», si veda Scorrano, L., Il Dante «fascista». Saggi, letture, note dantesche, Ravenna, Longo, 2001.

${ }^{3}$ Dionisotti, C., Geografia e storia della letteratura italiana, Torino, Einaudi, 1967, p. 279.
} 
fiorentino), una raccolta di studi in due volumi per quasi mille pagine, Dante e il suo secolo, con contributi, fra gli altri, di Carducci, Capponi, Tommaseo, Lambruschini, Guerrazzi, e infine la solenne cerimonia inaugurale con lo scoprimento della statua del poeta in una Piazza Santa Croce gremita di popolo (e aggiungiamoci, per incredibile coincidenza, la scoperta della cassetta contenente le Ossa Dantis nella chiesa di San Francesco a Ravenna il 27 maggio 1865).

La trasformazione di questo Dante poeta-vate investito di una missione nazionale in un conservatore irrimediabilmente prigioniero di una utopia regressiva, per dirla con Marco Santagata ${ }^{4}$, è stata repentina, considerando i tempi propri del dibattito critico: il nuovo paradigma si è imposto nell'arco di pochi anni, con un processo innescato verosimilmente da Benedetto Croce che con La poesia di Dante del 1921 interveniva a ridimensionare drasticamente la rilevanza della componente storico-ideologica della Commedia definendola, come si sa, allotria rispetto a quella più propriamente lirica $\mathrm{e}$ aprendo così la strada a una progressiva caduta di credito del Dante «politico» e «profetico». 5

Tenendo quindi presente l'egemonia di Croce sulla cultura italiana, ancora imperante nel corso degli anni ' 50 , e il nuovo clima determinato dalla caduta del fascismo e dall'imporsi dei valori antinazionalisti della neonata Repubblica, si capisce bene come dagli iniziali distinguo crociani si sia passati nel giro di pochi anni al ripudio di ogni connotazione patriottica di Dante, fino a giungere agli esiti estremi di un Dante reazionario così come viene definito in un celebre saggio di Edoardo Sanguineti:

Ma infine, non c'è bisogno di farla lunga con tutto quel sogno di un monarca universale che cavalchi la cavalcatura dell'umana specie, e su tutto lo spavento e lo sdegno di Dante per il puzzo dei villani che si sono inurbati nella sua Firenze, per capire che questo tardo discendente di Cacciaguida che piange da pieno paradiso la buona Firenze ordinata, sobria, pudica e pacifica dei vecchi tempi, è di buona razza reazionaria. ${ }^{6}$

Insomma, sembra proprio che la vulgata critica oggi dominante sia quella (di lontana ascendenza crociana, come si diceva) che separa la grandezza del poeta dalla

\footnotetext{
${ }^{4}$ Santagata, M., Dante. Il romanzo della sua vita, Milano, Mondadori, 2012, p. 16.

${ }^{5}$ «Appartengono al giro di queste indagini -allotrie, nel senso sopradetto- gli studi sulla filosofia di Dante e su quel tanto, se pur vi fu, che egli nel suo generale tomismo immise di altre correnti speculative o pensò di proprio; sul suo ideale politico, e le somiglianze e differenze che presenta verso altri ideali allora proposti e vagheggiati; sulle vicende della sua vita pubblica e privata, e il variare dei suoi concetti e speranze, e sulla cronologia delle opere e delle singole parti della Commedia in rapporto alle loro storiche occasioni; sull'eredità letteraria, classica e medievale, che egli accolse; su quanto egli conobbe della storia passata e della contemporanea; e su quel che credeva reale nei fatti a cui alluse, e su quel che stimava semplicemente probabile o addirittura immaginò pei suoi intenti; sull'allegoria generale e quelle particolari o incidentali del poema, e se il fine del poema sia etico-religioso o politico o entrambi questi fini combinati; e via enumerando e particolareggiando», Croce, B., La poesia di Dante, Bari, Laterza, 1921, p. 5.

${ }^{6}$ Sanguineti, E., Dante reazionario, Roma, Editori Riuniti, 1992, p. 284.
} 
pochezza dell'ideologo, la potenza suprema di chi è stato capace di «discriver fondo a tutto l'universo» dalla meschinità dell'analista sociale, tanto che si deve dare ragione a Lino Pertile quando, sottolineando questa contraddizione, afferma provocatoriamente che Dante oggi non viene più preso sul serio: «È il grande paradosso per cui si legge e si ama il sommo poeta, se ne riconosce e ammira il genio prodigioso, si gremiscono le piazze e i teatri in cui se ne recitano e spiegano i testi, ma non lo si prende sul serio». ${ }^{7}$

Ebbene, quello che si vorrebbe fare qui, invece, sarebbe proprio prendere Dante sul serio, tentando di sottrarlo alla tenaglia «reazionario» vs. «utopista» per individuare, se possibile, nel sistema ideologico dantesco una ratio che sia all'altezza della (o quanto meno si avvicini alla) grandezza del poeta.

Sembrerebbe a prima vista che per avviare una simile operazione, di carattere essenzialmente politico, il punto di partenza non possa essere appunto che politico, cioè un'analisi e una valutazione della dottrina dantesca dell'Impero contrapposta alle rivendicazioni comunali e autonomistiche che all'Impero si opponevano. Tuttavia una considerazione più attenta ci mostra che le posizioni politiche di Dante nascono da una riflessione fondata prima ancora che sugli assetti istituzionali, sui fenomeni socioeconomici del suo tempo: sono i risultati di questa analisi che impongono la restaurazione dell'Impero come l'unica contromisura in grado di impedire la degenerazione dei fenomeni economici in patologie sociali. È quindi da qui, dalla riflessione dantesca sulle strutture economico-sociali che si deve partire, facendo pernio su tre punti: la fenomenologia del mercante, la nozione di usura rispetto a quella di giusto profitto, il ruolo del denaro nella società mercantile. Ovviamente la mole di studi al riguardo è imponente, ma forse non del tutto esaustiva per quanto riguarda gli esiti e le reazioni che queste emergenze storiche provocarono nella personalità del poeta, e se si vuole nel suo stesso «romanzo familiare» (il padre, come si sa, era un cambiavalute).

Se dunque cerchiamo di ricostruire il percorso di formazione che portò Dante ad elaborare i suoi parametri di valutazione in merito a mercatura, usura e denaro non possiamo che partire da Brunetto Latini, l'intellettuale che, come dice Giovanni Villani, rese i fiorentini «accorti in bene parlare, e in sapere guidare e reggere la nostra repubblica secondo la Politica». ${ }^{8}$ Ora, è vero che il ruolo di «maestro di Dante» che la tradizione assegna a Brunetto va inteso a tutto campo, secondo quella missione di educatore della classe dirigente fiorentina che Brunetto si era assegnata con il Trésor prima, e poi con la Rettorica e il Tesoretto, e come del resto conferma Dante medesimo («ché 'n la mente m'è fitta, e or m'accora, / la cara e buona imagine paterna / di voi quando nel mondo ad ora ad ora / m'insegnavate come l'uom s'etterna»). Ma ci sembra fuor di dubbio che la traccia più profonda che Brunetto ha lasciato nella memoria di Dante non sia quella del sapere enciclopedico profuso a piene mani nel Trésor e nel

\footnotetext{
${ }^{7}$ Pertile, L., «Attualità di Dante», Studi Danteschi, 81 (2016), pp. 1-11, a p. 3.

${ }^{8}$ Villani, G., Nuova Cronica, IX, 10 (in Cronisti del Trecento, Milano, Rizzoli, 1935, p. 286).
} 
Tesoretto, svarianti dai bestiari all'astronomia, dalle scienze naturali alla geografia, dalla teologia alla mitologia, né tanto meno un generico magistero d'arte, ${ }^{9}$ quanto le riflessioni sulla società mercantile e borghese che in quegli anni stava prendendo forma a Firenze e che trova proprio nell'insegnamento di Brunetto la sua dignità culturale e il suo fondamento etico. È qui il cuore della lezione che Dante porterà a lungo con sé, certo per tutto il periodo fiorentino e anche dopo l'esilio, almeno fino alla rottura con la Universitas Alborum e con la compagnia malvagia e scempia. Brunetto, come conferma la citazione di Giovanni Villani, assume un ruolo pedagogico nei confronti di quelle forze popolari e borghesi di parte guelfa (e quindi anche del giovane Dante) che prendono il controllo di Firenze dopo Benevento e la fine degli Svevi e che ambiscono a unire alla potenza economica e alla forza militare anche una legittimazione etica e culturale. $\grave{E}$ soprattutto nel Tesoretto che viene avviata e perfezionata questa operazione di sgrossatura con cui si cerca di munire la nuova classe dirigente di artigiani, mercanti e banchieri di un codice di comportamento che permetta di distinguere il ricco dall'avaro, il giusto prezzo dalla speculazione, il credito dall'usura, la liberalità dallo sperpero, il dono dalla volgare ostentazione della ricchezza. Nei versi che seguono, per esempio, la polemica contro l'avarizia è mirata ad ottenere una civilizzazione della figura sociale del ricco mercante attraverso l'adozione dei canoni di larghezza e liberalità propri di quella nobiltà cortese soppiantata dalla nuova aristocrazia mercantile:

Ma colui ch'ha divizia
sì cade in avarizia
ché l'avere non spende
e già l'altrui non rende,
anz'ha paura forte
ch'anzi che vegna a morte
l'aver gli venga meno,
e pur ristringe il freno.
Così rapisce e fura
e dà mala misura
e peso frodolente
e novero fallente,
e non teme peccato
d'avistar suo mercato
né di commetter frode,
anzi si 'l tene in lode;
di nasconderlo sòle

${ }^{9} \mathrm{Si} \mathrm{ricordi}$ in proposito quanto è detto in DVE, I, 13: «Post hec veniamus ad Tuscos, qui, propter amentiam suam infroniti, titulum sibi vulgaris illustris arrogare videntur. Et in hoc non solum plebeia dementat intentio, sed famosos quamplures viros hoc tenuisse comperimus: puta Guictonem Aretinum, qui numquam se ad curiale vulgare direxit, Bonagiuntam Lucensem, Gallum Pisanum, Minum Mocatum Senensem, Brunectum Florentinum, quorum dicta si rimari vacaverit, non curialia, sed municipalia tantum invenientur». 


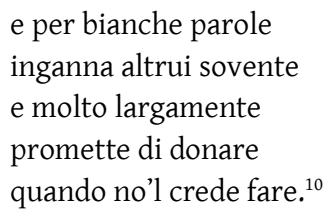

Il medesimo intento pedagogico teso alla defnizione di una morale civile rivelano i versi dedicati alla condanna dell'usura: «E un'altr'è, che non cura / di Dio e di Natura / sì doventa usoriere / e in molte maniere / ravolge suo' danari / che li son molto cari; / non guarda die né festa, / né per pasqua non resta, / e non par che li 'ncresca / pur che moneta cresca». ${ }^{11}$

Tuttavia in determinate circostanze l'usura può rivelarsi un male necessario: «Ancora abbi paura / d'improntare ad usura; / ma, se pur ti convene / aver per spender bene, / prego che 'l rende avaccio, / ché nonn'è bel procaccio / né piacevol convento / di diece render cento: / già d'usura che dai, / nulla grazia non hai; / ne'ciò non ha larghezza, / ma tua grande pigrezza». ${ }^{12}$

Dunque, ci dice Brunetto che sarebbe meglio evitare di prendere soldi in prestito, ma «se pur ti convene», cioè se ti è necessario, almeno impiega il denaro spendendolo bene, ossia senza cadere nell'avarizia e con investimenti che vadano a vantaggio della comunità, e inoltre cerca di restituirlo alla svelta per evitare interessi troppo pesanti. Qui sembra proprio che Brunetto stia descrivendo con grande lucidità e spirito pratico una sorta di «economia politica» fondata su una rapida circolazione del denaro e sulla condanna della tesaurizzazione bollata come avarizia e pigrizia: viene in mente l'evangelica parabola dei talenti, in cui il servo che non ha investito il denaro ricevuto dal padrone viene condannato per la sua pigrizia, cioè perché tesaurizza senza contribuire alla circolazione della ricchezza. ${ }^{13}$

Quanto al denaro, Brunetto nel Tresor sembra considerarlo come uno strumento fondamentalmente neutro («justice sans ame») che ha in sé la capacità di uniformare la realtà adeguandola a un comune criterio di valore: «Por ce fu deniers trovés premierement, por çou k'il igaillauent les choses ki desigaus estoient; et deniers est aussi come justice sans ame, por k'il est un mi par quoi les choses desigaus tornent a

\footnotetext{
${ }^{10}$ Latini, B., Il Tesoretto, ed. M. Ciccuto, Milano, Rizzoli, 1985, vv. 2753-2774.

${ }^{11}$ Ibid., vv. 2787-2796.

${ }^{12}$ Ibid., vv. 1511-1522.

${ }^{13}$ «Accedens autem qui unum talentum acceperat, ait: -Domine, novi te quia homo durus est: metis, ubi non seminasti, et congregas, ubi non sparsisti; et timens, abii et abscondi talentum tuum in terra. Ecce, habes, quod tuum est- Respondens autem dominus eius dixit ei: -Serve male et piger! Sciebas quia meto, ubi non seminavi, et congrego, ubi non sparsi? Oportuit ergo te mittere pecuniam meam nummulariis, et veniens ego recepissem, quod meum est cum usura. Tollite itaque $a b$ eo talentum et date ei, qui habet decem talenta: omni enim habenti dabitur, et abundabit; ei autem, qui non habet, auferetur ab eo», Mt. 25, 24-29.
} 
igaillité». ${ }^{14}$

E infine, in questa sintetica campionatura non poteva mancare l'apologia del dono: «E se cosa adivenga / che spender ti convenga, / guarda che sia intento / sì che non paie lento: / ché dare tostamente / è donar doppiamente, / e dar come sforzato / perde lo dono e 'l grato». ${ }^{15}$ Inevitabile il rimando a Dante, Conv., I, 8:

La terza cosa, ne la quale si può notare la pronta liberalitade, si è dare non domandato: acciò che 'l domandato è da una parte non vertù ma mercatantia, però che lo ricevitore compera, tutto che 'l datore non venda. Per che dice Seneca che «nulla cosa più cara si compera che quella dove i prieghi si spendono». Onde a ciò che nel dono sia pronta liberalitade e che essa si possa in esso notare, allora, se conviene esser netto d'ogni atto di mercatantia, conviene esser lo dono non domandato.

Ancora all'altezza del Convivio, dunque, Dante mostra di condividere la lezione di pedagogia civile di Brunetto e in genere degli intellettuali della generazione precedente (per esempio Bono Giamboni), con la differenza che mentre costoro si rivolgevano alla borghesia mercantile, nel Convivio Dante si rivolge in primo luogo (anche se non esclusivamente) alla nobiltà («principi, baroni, cavalieri, e molt'altra nobile gente, non solamente maschi ma femmine, che sono molti e molte in questa lingua volgari e non litterati», I, 9).

Sembra dunque emergere una fisionomia dantesca tutt'altro che reazionaria e rivolta a uno sterile rimpianto del passato, ma che configura, al contrario, l'immagine di un uomo pienamente inserito nel suo tempo e consapevole delle trasformazioni che proiettano irreversibilmente Firenze in una dimensione oramai lontanissima dalla città racchiusa nella «cerchia antica». Compito dell'intellettuale sarà dunque non quello di scagliare anatemi, ma di impegnarsi per civilizzare questa nuova realtà, per rendere questa nuova ispida razza padrona fatta di mercanti, cambiatori, artigiani, banchieri, capace di «bene parlare» e di «sapere guidare e reggere la nostra repubblica secondo la Politica». È questa la missione che Dante si assegna e di cui sente tutta la responsabilità soprattutto dopo la quasi contemporanea scomparsa di Brunetto (1294) e di Bono Giamboni (1292), i due intellettuali che prima di lui si erano assunti il compito di presiedere all'educazione civile dei fiorentini. È una missione che può essere svolta, però, solo se preliminarmente viene fatta chiarezza sulla questione della mercatura, del profitto e del credito a interesse che secondo una ricostruzione posteriore, tanto diffusa quanto imprecisa, erano considerate irrevocabilmente forme di usura e quindi oggetto di una condanna indiscriminata. In realtà non è così, né poteva esserlo da parte di una élite intellettuale che, pur obbligata ai canoni rigorosi della teologia morale, restava comunque parte integrante di una società da cui non poteva prendere le distanze in maniera radicale, pena l'irrilevanza e l'autoemarginazione. Come scrive esemplarmente Armando Sapori a proposito di San Tommaso, non è pensabile che una

${ }^{14}$ Latini, B., Li livres dou tresor, ed. F. J. Carmody, Genève, Slatkine Reprints, 1998, p. 199.

${ }^{15}$ Ibid., vv. 1413-1420. 
cultura che si propone come guida spirituale (e non solo) di un'intera società si distacchi volontariamente da quella realtà che intende dirigere. ${ }^{16}$ Dante se ne rende perfettamente conto e pur scagliandosi contro l'avarizia, la cupidigia e il «maladetto fiore» si guarda bene dal coinvolgere in una condanna indiscriminata i protagonisti della vita economica e sociale del suo tempo. È una posizione che trova il suo fondamento dottrinale proprio nelle enunciazioni tomistiche riguardo al profitto ${ }^{17}$ alla mercatura, ${ }^{18}$ al giusto prezzo,${ }^{19}$ dove il parametro in base al quale misurarne il grado di ammissibilità è la corrispondenza a quell'ideale di medietas che sta alla base dell'Etica aristotelica ${ }^{20}$ e che da lì passa poi nella morale cristiana, smussandone le punte più ascetiche e rigoriste. San Tommaso è a questo proposito chiarissimo: «In quibuscumque bonum consistit in debita mensura, unde necesse est quod per excessum vel deminutionem illius mensurae malum proveniat: in omnibus, autem, bonum consistit in quadam mensura». ${ }^{21}$

La stessa tradizione francescana, sovente indicata come alternativa radicale a

${ }^{16}$ «Naturalmente [Tommaso] non poteva essere insensibile al fermento che si agitava intorno a lui, non poteva chiudere gli occhi allo spettacolo grandioso che gli si parava dinanzi: una corsa infrenabile verso un generale elevamento della vita per mezzo del moltiplicarsi delle imprese costituite per il guadagno. Ché, si ammetta pure, e ne conveniamo senz'altro, che egli abbia tentato in nome dei principi cristiani di costituire una remora al dilagare di sentimenti che dovevano apparire quasi un risorgere del paganesimo; ma non si potrà credere che egli abbia voluto rendere volontariamente inefficace il suo ammaestramento con lo staccarlo dalla realtà», Sapori, A., Studi di storia economica medievale, Firenze, Sansoni, 1940, p. 111. Una risorsa bibliografica preziosa sui temi dell'usura e del prestito a interesse è Barile, N.L., «Credito, usura, prestito a interesse», Reti medievali, 11 (2010), pp. 475-504.

17 «Lucrum tamen, quod est negotiationis finis, etsi in sui ratione vitiosum vel virtuti contrarium: unde nihil prohibet lucrum ordinari ad aliquem finem necessarium vel etiam honestum: et sic negotiatio licita reddetur, sicut cum aliquis lucrum moderatum, quod negotiando quaerit, ordinat ad domus suae substentationem vel etiam ad subveniendum indigentibus», Tommaso d'Aquino, Summa theologiae II, 2, quest. LXXVII, art. 4.

${ }_{18}$ «Nec tamen negotiatores omnino a civitate oportet excludi, quia non de facile potest inveniri locus, qui sic omnibus vitae necessariis abundet, quod non indigeat aliquibus aliunde allatis [...] Unde oportet quod perfecta civitas moderate mercatoribus utatur», Tommaso d'Aquino, De regimine principum IV, 23.

${ }_{19}$ «Ideo dico, quod iustum pretium rerum non est punctualiter determinatum, sed magis in quadam aestimatione consistit; ita quod modica additio vel minutio non videtur tollere aequalitatem iustitiae», Tommaso d'Aquino, Summa theologiae II, 2, quest. LXXVII, art.1.

${ }^{20}$ «Est ergo virtus habitus electivus in medietate existens que ad nos determinat racione, et ut utique sapiens determinabit. Medietas autem duarum maliciarum, huius quidem secundum habundanciam, huius autem secundum defectum; et adhuc huic, has quidem deficere, has autem superabundare $a b$ eo quod oportet et in passionibus et in operacionibus, virtutem autem medium et invenire et eligere. Propter quod secundum substanciam quidem et racionem quid est esse dicentem, medietas est virtus, secundum optimum autem et bene, extremitas», Aristotele, Ethica Nicomachea, II, 5, in Aristoteles latinus, XXVI, Leiden, ed. R.A. Gauthier, 1973, p. 404 (versione latina di Roberto Grossatesta).

${ }^{21}$ Tommaso d'Aquino, Summa theologiae II, 1, quest. CXVIII, art.1. 
un'interpretazione «moderata» del rapporto con la ricchezza e i beni materiali, in realtà è molto meno oltranzista di quanto suggerisca la leggenda ascetica degli Spirituali. È proprio uno dei maestri degli Spirituali, Pietro di Giovanni Olivi (che fu lettore a Firenze nello Studio di Santa Croce dal 1287 al 1289, e fu verosimilmente ascoltato da Dante) a proporre in un suo trattato, cruciale per capire il rapporto dell'ala più pauperista del cristianesimo medievale con i meccanismi della società mercantile, una interpretazione priva di condanne di principio contro le pratiche del commercio, del profitto, del credito e della mercatura, che vengono anzi considerate lecite se mantenute all'interno di una medietas che le liberi dalla connotazione di «avarizia» e «cupidigia». L'Olivi, per esempio, discute la questione se il mercante possa guadagnare lecitamente, rispondendo in modo affermativo:

Circa venditionum et emptionum contractus queramus primo an res possint licite et absque peccato vendi plus quam valeant vel minus emi. Et videtur quod sic. Quia aliter fere tota communitas vendentium et ementium contra iustitiam peccarent, quia forte omnes volunt care vendere et vile emere. Item licitum est mihi rei mee precium ponere quod volo, nec aliquod ius me compellit me dare aut commutare res meas absque pretio mihi placito et a me pretaxato; sicut e contrario nullus compellitur vel cogitur rem alterius emere ultra pretium sibi placitum. Si igitur contractus vendendi et emendi est mere voluntarius, igitur taxatio pretii rerum venalium erit mere voluntaria, ac per consequens sortietur ius et forum mere voluntarium. Iuxta illud vulgare verbum: Tantum valet res, quantum vendi potest- Item secundum ordinem iuris et iustitie et caritatis commune bonus prefertur et preferri debet bono privato, sed communi saluti hominum post lapsum expedit quod taxatio pretii rerum venalium non sit punctualis nec secundum valorem absolutum rerum, sed potius ex comuni consensu utriusque partis vendentium, scilicet, et ementium libere pretaxetur. Hoc enim minore periculo fraudum includit. Illud vero posset fieri absque peccato, quia punctualis et absolutus valor rerum vix alicui patet certitudinaliter ad plenum. ${ }^{22}$

In termini più generali, l'olivi argomenta poi che la mercatura e il profitto non costituiscono di per sé una prassi peccaminosa, ${ }^{23}$ e addirittura concede una patente di

${ }^{22}$ Pietro di Giovanni Olivi, Tractatus de emptionibus et venditionibus, de usuris, de restitutionibus, ed. G. Todeschini, Roma, Istituto Storico Italiano per il Medio Evo, 1980, p. 51.

${ }^{23} \mathrm{Ibid}$., pp. 62-63: «Queritur an emens rem quamcumque ut eam immutatam et immelioratam pro maiori pretio vendat, sicut communiter faciunt mercatores, peccet in hoc mortaliter aut saltem venialiter. Dicendum quod non est necesse hic directe et per se includi peccatum, quamvis hoc sit valde rarum et difficile. Quod hoc autem de se sit licitum triplici ratione et duplici testimonio et auctoritate probetur. Prima autem ratio est ex manifestis commodis et necessitatibus provenientibus comunitati ex actu et offitio mercandi. Et similiter cum hoc ex honerosis laboribus ac periculis et expensis et industriis et pervigilibus providentis que exigunt offitium illud. Constat enim quod multa desunt uni urbi vel patrie que habundant in altera. Rursus mercatores suas pecunias et etiam personas ac deinde merces suas ex pecunia emptas multis periculis exponunt; nec sunt certi an de mercibus emptis suum rehabeant capitale. Si etiam non essent industrii in rerum valoribus et pretiis et commoditatibus subtiliter extimandis, non essent 
liceità al prestito a interesse $\mathrm{e}^{24}$ (saranno del resto proprio i francescani, in polemica con i domenicani, a promuovere l'istituzione dei montes pietatis come agenzie di credito a interesse minimo per i poveri) ${ }^{25} \mathrm{E}$ infine la stessa figura di san Francesco, se la liberiamo dall'alone agiografico (Tommaso da Celano, San Bonaventura, i Fioretti, il Sacrum commercium sancti Franciscicum domina paupertate e la stessa ricostruzione biografica che ne fa Dante in Par. XI), rivela tratti sorprendenti di vicinanza, se non di integrazione, con i valori fondanti della società cortese, fatti propri in quello stesso giro di anni dalla nascente civiltà mercantile. Il primo ad accorgersene fu Pio Rajna, che in un breve ma acutissimo articolo del 1926 scrive a proposito del Santo di Assisi :

Ci è attestata l'eleganza del vestire, spinta fino alla stravaganza; è da supporre senza titubare destrezza negli esercizi del corpo, e senz'armi e con le armi [...] Tutto ravvicinando e sommando, egli, nel fiore della gioventù, ci appare come un cavaliere al quale manca soltanto d'essere fatto cavaliere. E ad esser fatto egli anela. [...] Francesco volse ad altra meta la prora. Ma l'animo suo rimase sempre il medesimo. Liberale, portò la liberalità all'estremo limite, non volendo possedere nulla. Gaio, continuò ad essere gaio. Amante dei canti, seguitò a cantare, e a farlo nel linguaggio in cui certo aveva cantato preferibilmente anche prima, e che aveva avuto tanta parte nella formazione del suo spirito. E i paladini di Carlo Magno e i compagni di Artù seguitarono a vivere nella sua fantasia. ${ }^{26}$

Del resto, le connotazioni cavalleresche di Francesco sono gà presenti nella duecentesca Legenda trium sociorum ${ }^{27}$, e più recentemente sono state recuperate e

ad hoc idonei. Item, nisi essent honorabiles et fide digni, non eis a diversarum terrarum gentibus prout expedit huic offitio crederetur. Si etiam non essent pecuniosi non possent grandes et caras merces prout terris expedit providere. Ex his autem aperte concluditur quod lucrum predictis circumstantiis competens inde possint et debent reportare. Ex quo ulterius sequitur quod usque ad aliquam mensuram congruam possunt suarum mercium pretium augere».

${ }^{24} \mathrm{Ibid}$., p. 67: «Queritur an pro mutuo prestito accipere plus quam mutuatum est, sit contra ius naturale et divinum. Et videtur quod non, quia constat quod mutuatio et res prestita mutuo aliquam commoditatem confert illi cui prestatur. Ergo secundum naturalem equitatem potest huiusmodi commoditas pretio extimari et ricompensari».

${ }^{25}$ Cfr. Noonan, J.T., Prestito professionale e istituzionale, in O. Capitani (ed.), L'etica economica medievale, Bologna, Il Mulino, pp. 189-208.

${ }^{26}$ Rajna, P., «S. Francesco d'Assisi e gli spiriti cavallereschi», Nuova Antologia, 1, 1926, pp. 3-19, a p. 9.

${ }^{27}$ «Hic, postquam fuit adultus et subtilis ingenii factus, artem patris id est negociationem exercuit, sed dissimiliter valde quoniam ipso hilarior et liberalior, deditus iocis et cantibus, civitatem Assisii die noctuque circumiens sibi similibus sociatus, in expendendo largissimus adeo ut omnia quae habere poteret et lucrari in comestionibus aliisque rebus consumeret. Propter quod multotiens arguebatur a parentibus dicentibus ei quod tam magnas expensas in se et in aliis, ut non eorum filius sed cuiusdam magni principis videretur. Quia tamen divites erant parentes eius et ipsum tenerrime diligebant, tolerabant eum in talibus ipsum turbare nolentes. Mater autem eius, cum de prodigalitate sua sermo a convicinis fieret, respondebat: -Quid de filio meo putatis? Adhuc erit filius Dei per gratiam- Ipse vero non solum in his erat largus, immo prodigus, sed etiam in indumentis multipliciter excedebat, cariores pannos faciens quam ipsum deceret habere. Erat 
sottolineate da Franco Cardini ${ }^{28}$ a smentita di una interpretazione del francescanesimo rigidamente ascetica e fieramente ostile a ogni forma di temperamento del pauperismo attraverso la legittimazione morale del mercante, riscattato dal peccato di usura attraverso la pratica dello justum pretium (come già abbiamo visto in San Tommaso e in Pietro di Giovanni Olivi). E poiché bisogna pur vivere, la dottrina francescana non manca di indicare nel lavoro il modo con cui la gente comune, non chiamata alla testimonianza di un cristianesimo radicale come lo sono gli ordini mendicanti, deve provvedere al proprio mantenimento. ${ }^{29}$ Sempre sul versante di una tolleranza in nome della medietas va collocato il giudizio di condanna che Dante fa pronunciare a San Bonaventura contro Ubertino da Casale e Matteo d'Acquasparta che forzano in direzioni opposte la regola francescana, sì «ch'uno la fugge e l'altro la coarta» (Par. XII, 126).

Dunque, dall'esperienza fiorentina di Dante sembrerebbe emergere nei confronti della borghesia mercantile non un rifuto radicale, ma un atteggiamento pedagogico tendente a smussarne le asperità utilitaristiche e a ricondurla ai canoni dell'etica cortese. Va precisato tuttavia che le fonti citate fin qui, a cui Dante avrebbe potuto attingere per elaborare questa sua impresa pedagogica, sono prive di riscontri diretti, atteso che del processo di formazione intellettuale del poeta durante la giovinezza fiorentina sappiamo ben poco: ${ }^{30}$ al di là delle «scuole de li religiosi e delle disputazioni de li filosofanti» (Conv., II, 12), resta accertato solo il ruolo di Brunetto in quella chiave di nuova «etica sociale» a cui si è fatto fin qui riferimento e che è stata studiata da Enrico Fenzi in pagine esemplari dedicate appunto al rapporto fra il Tesoretto e la canzone Poscia ch'amor. ${ }^{31}$ Per il resto, restiamo nel campo delle ipotesi. È in ogni caso significativo che nelle due canzoni dottrinali, Le dolci rime e Poscia ch'amor, scritte fra il 1295 e il 1296, cioè poco dopo la morte di Brunetto (1294) e contemporaneamente all'avvio della carriera politica di Dante (iscrizione alla corporazione dei medici e speziali, 1295), il tema affrontato sia appunto quello dell'educazione civile della nuova classe dirigente fiorentina sulla scorta della lezione di Brunetto. Come giustamente nota Enrico Fenzi,

enim quasi naturaliter curialis in moribus et in verbis», Legenda trium sociorum, in E. Menestò, e S. Brufani, (eds.), Fontes franciscani, Assisi, Porziuncola, 1996, pp. 1375-1376.

${ }^{28}$ Cardini, F., Francesco d'Assisi, Milano, Mondadori, 1991.

${ }^{29}$ Cfr. in proposito Manselli, R., Evangelismo e povertà, in Capitani, (ed.), La concezione della povertà nel Medioevo, op. cit., pp. 190-208.

${ }^{30}$ Secondo Zygmunt Baranski, ricostruire la Bildung del poeta è impossibile, perché Dante fu un eclettico, un «raccoglitore di frammenti privo di una formazione intellettuale di scuola, un grande amateur che amalgamò differenti tradizioni in una sintesi originale», Baranski, Z., «Sulla formazione intellettuale di Dante», Studi e problemi di critica testuale, 90 (2015), pp. 31-53. Sullo stesso tema vedi anche Romanelli, M., «La musica nella Commedia: un isomorfismo della lingua perfetta», Studi Italiani, 29/1 (2017), pp. 5-29, alle pp. 26-27.

${ }^{31}$ Cfr. Fenzi, E., «Sollazzo e leggiadria. Un'interpretazione di Poscia ch'amor del tutto $m$ 'ha lasciato», in Id., Le canzoni di Dante. Interpretazioni e letture, Firenze, Le Lettere, 2017, pp. 263-336, alle pp. 280-283. 
l'implicito omaggio verso una delle voci più autorevoli che per tanti anni aveva accompagnato la vita politica della città, sembra davvero costituire un passo obbligato per Dante che esce dallo stilnovo e dal suo elitario ambito di amicizie e cultura e che trasforma la sua poesia in strumento di dibattito nel campo dell'etica sociale, e finalmente comincia la propria attività politica negli organi di governo del Comune. ${ }^{32}$

Come si sa, Le dolci rime è la canzone commentata nel quarto libro del Convivio e dedicata a definire la nozione di nobiltà. Il tema non è nuovo: già nella lirica provenzale la superiorità della nobiltà dell'animo sulla nobiltà per nascita è un topos largamente diffuso, ma limitato al contesto della fin'amor, come documenta Erich Köhler. ${ }^{33}$ Dante però supera i confini del dibattito interno alla cultura cortese e si rivolge a un interlocutore collettivo che è, come già si è detto, la borghesia mercantile e finanziaria, la nuova classe dirigente della società comunale. Il nucleo dell'argomentazione dantesca riguarda il valore morale che si deve dare alla ricchezza: le divizie non sono in rapporto diretto con la nobiltà, perché, contrariamente a quello che si crede, «non posson gentilezza dar né tòrre / però che vili son da lor natura», e questa natura vile delle ricchezze appare nel fatto che esse, «quantunque collette, / non posson quietar, ma dàn più cura; / onde l'animo ch'è dritto e verace / per lor discorrimento non si sface». Anche l'argomento della natura bassamente materialistica della ricchezza, che con una frustrante progressio ad infinitum non riesce mai a conseguire l'appagamento del desiderio ed è quindi origine di una continua e crescente insoddisfazione, non è nuovo: e del resto è proprio Dante, nel commento alla canzone, a indicare le proprie fonti facendo riferimento a Cicerone, Salomone, Boezio, Seneca, Orazio, Giovenale e alle Scritture. ${ }^{34}$ Quello che invece è frutto di un'elaborazione originale è la descrizione delle

\footnotetext{
${ }^{32}$ Ibid., p. 283.

${ }^{33}$ «Il servizio d'amore non è altro che la possibilità di acquistare pretz e onor, cioè prestigio e onore, che dipendono non più dalla proprietà ma dai rapporti sociali, è uno strumento di perfezionamento; per i trovatori esso diventa un fatto pubblico e un principio supremo. Con il servizio d'amore la piccola nobiltà riesce ad imporre come essenza della nobiltà una forma di vita a lei congeniale e fa del proprio onore l'onore di tutto lo «stato». La partecipazione del potente si giustifica solo con la liberalità, che diventa il criterio per giudicare se egli è veramente disponibile per realizzare la missione storica della nobiltà. Il ricco avaro è la rovina di tutto lo "stato« cavalleresco, che la piccola nobiltà si sente chiamata a rappresentare nella sua totalità e che ritiene di dover difendere e mantenere. Il compimento di questa missione universale, che è affidato all'amante cortese, si impone ora come misura di ogni valore umano al posto del rango e della ricchezza: è il trionfo della nobiltà dell'animo», Köhler, E., Sociologia della fin'amor, Padova, Liviana, 1976, pp.160-161.

${ }^{34}$ «E queste tutte parole sono di Tullio, e così giacciono in quello libro che detto è. E a maggiore testimonianza di questa imperfezione, ecco Boezio in quello De Consolatione dicente: -Se quanta rena volve lo mare turbato dal vento, se quante stelle rilucono, la dea della ricchezza largisca, l'umana generazione non cesserà di piangere- E perché più testimonianza, a ciò ridurre per pruova, si conviene, lascisi stare quanto contra esse Salomone e suo padre grida; quanto contra esse Seneca, massimamente a Lucillo scrivendo; quanto Orazio, quanto Iuvenale e, brievemente, quanto ogni scrittore, ogni poeta; e quanto la verace Scrittura divina chiama contra queste false meretrici, piene di tutti defetti», Conv., IV, 12.
} 
forme che la nobiltà assume nelle diverse fasi della vita, sviluppata nella settima stanza:

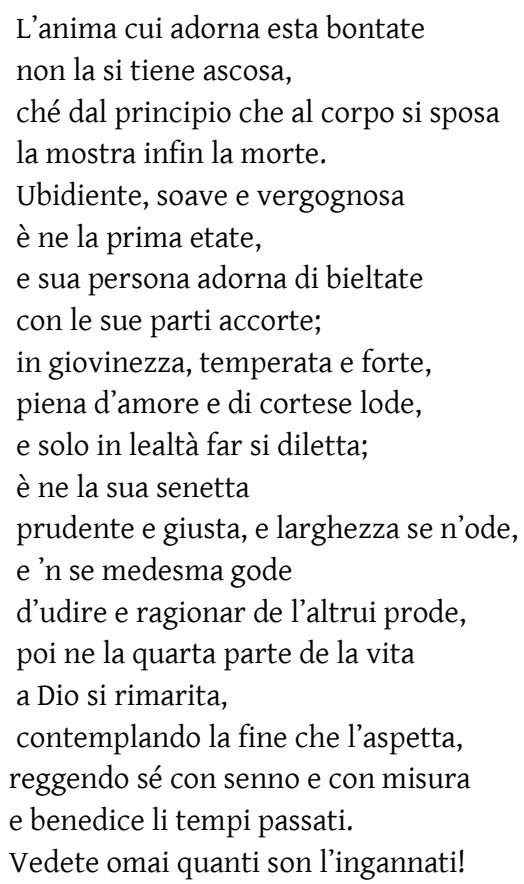

Siamo con ogni evidenza di fronte a una sorta di «galateo sociale» che specifica $\mathrm{i}$ comportamenti propri dell'uomo dotato di "gentilezza», e benché in chiusura si ammetta che molti sono «l'ingannati», resta comunque l'impegno a operare una demistificazione della falsa nobiltà e la fiducia che sia possibile restaurare e diffondere quel «valore, / per lo qual veramente omo è gentile»: la strada verso il recupero di una corretta etica sociale appare ancora aperta.

Certo, si tratta di un cammino arduo, perché la maggioranza è schiava di una falsa immagine della nobiltà collegata alla ricchezza («Vedete omai quanti son l'ingannati!»), e soprattutto perché di questa maggioranza «ingannata» fanno parte non solo gli uomini «grossi» ma anche uomini insigni per censo, sensibilità e magistero culturale.

L'altra grande canzone dottrinale di ambito fiorentino è, come abbiamo detto, Poscia ch'amor, risalente probabilmente al 1296. Se Le dolci rime fondava la teoria della nuova nobiltà, Poscia ch'amor ne definisce attraverso una dettagliata casistica la pratica sociale e i comportamenti concreti attraverso la definizione del concetto di leggiadria. ${ }^{35}$

${ }^{35}$ Per comprendere la portata polemica della canzone è essenziale notare che la nozione di leggiadria così come viene qui proposta da Dante assume un valore innovativo rispetto a quello che il termine rivestiva nella tradizione provenzale da cui proviene nella forma originaria di leujairia, «leggerezza», nel senso dell'attitudine a un'eleganza frivola che si associa agli aspetti più deteriori della vita mondana. 
Subito nella prima stanza Dante enuncia con la massima chiarezza il suo fine, quello cioè di smascherare chi occulta la propria viltà morale sotto una nozione distorta della leggiadria (7-14): «i' canterò così disamorato / contra 'l peccato, / ch'è nato in noi, di chiamare a ritroso / tal ch'è vile e noioso / con nome di valore, / cioè di leggiadria, ch'è bella tanto / che fa degno di manto / imperial colui dov'ella regna».

A questa dichiarazione di intenti segue una dettagliata casistica dei falsi leggiadri, in verità vili e noiosi: primi, quelli che sperperano le loro «divizie» con la volgarità degli arricchiti esibizionisti presumendo così di essere annoverati fra i nobili («credon potere / capere là dove li boni stanno», 21-22), ma si sbagliano perché sarebbe meglio per loro se imparassero ad amministrare più saggiamente il loro denaro; poi ci sono quelli che scambiano la leggiadria con la gola, con la lussuria, con l'ostentazione di eleganza nel vestire, con la vuota brillantezza della conversazione, con l'assumere pose affettate, con il prendere «villan diletto» (54) dall'amore inteso come volgare sensualità che li rende simili a «animai sanza intelletto» (57).

Ora, se Dante fosse, come oggi molti ancora ritengono, un conservatore chiuso e incapace di proporre un progetto diverso da quello della restaurazione del passato, si limiterebbe a rappresentare questa galleria degli orrori sociali come motivazione di una condanna senza appello del presente; invece, dopo la denuncia, segue la proposta fondata appunto su una corretta interpretazione della 'leggiadria' attraverso la quale passa il riscatto civile della nuova classe dirigente, evidentemente ritenuto ancora possibile per quanto arduo («non so cui» recita il testo, cioè «non so bene a chi mi rivolgo», tanto appare dubbio che ci siano ancora spiriti così nobili da essere sensibili all'argomento). Ecco dunque che, a partire dalla quarta stanza, si esplicita il proposito di definire la vera leggiadria che è costituita dalla sintesi di tre componenti: amore, sollazzo e opera perfetta, cioè amore per la sapienza, senso gioioso del vivere e perfezione virtuosa dell'operare («Sollazzo è che convene / con esso Amore e l'opera perfetta: / da questo terzo retta / è vera leggiadria», 89-92).

La canzone si chiude con un verso che esprime lo scoraggiamento di Dante nel constatare che la realtà procede in tutt'altra direzione («Color che vivon fanno tutti contra»), e tuttavia, pur nell'amaro pessimismo, l'attitudine pedagogica volta a una possibile palingenesi sociale resta intatta. ${ }^{36}$

È dunque con questo dichiarato intento riformatore che Dante si colloca all'interno del Comune mercantile: nessuna fuga nel passato, quindi, nessuna chiusura antistorica e reazionaria, ma al contrario un preciso progetto riformatore portato avanti con

${ }^{36}$ Osserva ancora Enrico Fenzi che «la lamentela cessa dunque d'esser tale, e diventa momento di scontro, di attacco, di apertura al futuro. Se Color che vivon fanno tutti contra, con tanta più forza occorrerà che un siffatto degradato presente debba essere condannato, per riportare il nome di valore della leggiadria entro le occasioni e le necessità del vivere civile: nelle dottrinali, questo è l'impegno del momento, e la grande scommessa intellettuale e morale per l'avvenire», Fenzi, Le canzoni di Dante. Interpretazioni e letture, op. cit., p. 336. 
decisione pari alla coscienza della difficoltà del compito: verrebbe da dire, gramscianamente, con il pessimismo dell'intelligenza e l'ottimismo della volontà. Ci troviamo di fronte, insomma, a una concezione «moderata» e «riformista» della società mercantile, del profitto, del denaro ecc.: un universo osservato con diffidenza e con forte spirito critico, ma non oggetto di anatema e di damnatio in nome di un passato idealizzato e rimpianto. È ancora operante, con ogni evidenza, l'approccio equilibrato e tollerante rispetto alle questioni del denaro, della mercatura e del profitto che abbiamo verificato nella tradizione tomistica e addirittura nel pauperismo francescano e, per quanto riguarda specificamente l'ambiente fiorentino, nella minuziosa precettistica di Brunetto tesa a fondare, parrebbe, quasi un embrione di economia politica. ${ }^{37}$

Su questi propositi di impegno civile volto a una acculturazione in senso nobile e leggiadro dei ceti dirigenti fiorentini piomba improvvisamente la catastrofe dell'esilio: fra il gennaio e il marzo del 1302 Dante subisce due condanne, la prima al confino e a una pena pecuniaria, la seconda a morte. Nonostante si getti subito nella battaglia per rovesciare le sorti politiche della parte bianca e sue personali (il convegno di Gargonza, l'organizzazione della Universitas Alborum di cui viene nominato cancelliere, i progetti di iniziative militari), le speranze del poeta si rivelano presto illusorie: la sanzione definitiva è data dal rovescio della Lastra (1304), dove i fuorusciti bianchi vengono disastrosamente respinti nel loro tentativo di rientrare a Firenze con la forza delle armi ${ }^{38}$ Ma Dante aveva ormai già dall'anno precedente rotto i ponti con la «compagnia malvagia e scempia» dei fuorusciti e preso a fare parte per se stesso, probabilmente, come sostiene Mirko Tavoni sulla scorta delle informazioni fornite dai commenti dell'Ottimo ${ }^{39}$ e di Benvenuto, ${ }^{40}$ in seguito alla sanguinosa rotta di Castel Puliciano (1303).

${ }^{37} \mathrm{Si}$ veda quanto scrive in proposito Antonio Montefusco: «In definitiva, Brunetto Latini forse inventa (oppure riporta in poesia) una economia politica cristiana. La definisce e la include nel suo grande progetto pedagogico cittadino. La propone come misura di giudizio sul mondo del Comune. La integra nel suo tentativo di rendere «socievoli» gli aggressivi «cavalieri». Indica loro la possibilità di adeguarsi al bene della collettività. Nel farlo indica, un po' nascostamente e quasi delicatamente, il modello del buono e vero mercante: che non deve essere avaro, deve sapere spendere bene, deve trasformare la spesa in dono. È un'operazione straordinaria, ma soprattutto è un'operazione di successo, perché la cultura «popolare « fiorentina degli anni successivi se ne dimostrerà per sempre impregnata», Montefusco, A., Banca e poesia al tempo di Dante, Milano, ASSB, 2017, p. 29.

${ }^{38}$ Sull'argomento la migliore trattazione a mia conoscenza è quella di Mirko Tavoni in Qualche idea su Dante, Bologna, Il Mulino, 2015, pp. 110-146.

${ }^{39}$ «E quel che più ec. Dice, che la mala compagnia di quelli della sua setta, con li quali elli cadrà, la quale è tutta ingrata verso Iddio delli ricevuti beneficii, tutta matta per soperchia prosperitade, ed empia senza pietate, li graverà più ch'altro. E dice, ch'essa si farà contra lui, la qual cosa divenne quando elli sè oppose, che la detta Parte Bianca cacciata di Firenze, e già guerreggiante, non richiedesse li amici il verno di gente, mostrando le ragioni del piccolo frutto; onde poi, venuta la state, non trovarono l'amico com'elli era disposto il verno; onde molto odio ed ira ne portarono a Dante; onde elli si partì da loro» (Ottimo commento, prima redazione, Dartmouth Dante Project, p. 2839).

${ }^{40}$ «Et subdit quod cito sequetur vindicta de hoc; unde dicit: ma poco appresso, quia scilicet tertio 
È in questo quadro che si colloca la definitiva rinuncia al grande progetto riformatore della borghesia fiorentina, sostituito da una fase di smarrimento e di angosciosa impotenza (fase non lunga, perché la rassegnazione e la resa non appartenevano alla personalità dantesca: e infatti già nel corso del 1304 egli riprende la stesura del Convivio assegnandosi quindi una meta ancora più ambiziosa, cioè la organizzazione di una classe dirigente non più limitata all'ambito fiorentino, ma estesa alla comunità dei «Latini»). Tuttavia è innegabile che una fase di smarrimento ci sia stata: ne è drammatica testimonianza la possente canzone Doglia mi reca, che rappresenta una vera palinodia rispetto alla prospettiva riformatrice espressa dalle due dottrinali degli anni '90. Qui, nella chiusa angoscia che lo domina di fronte alla inemendabilità della corruzione umana, Dante attinge a esiti che potremmo definire nichilistici: tale si deve considerare l'appello finale rivolto alle donne alle quali viene imposto di rinunciare all'amore poiché amare, in una società dominata dai malvagi, significa rendersi loro complici:

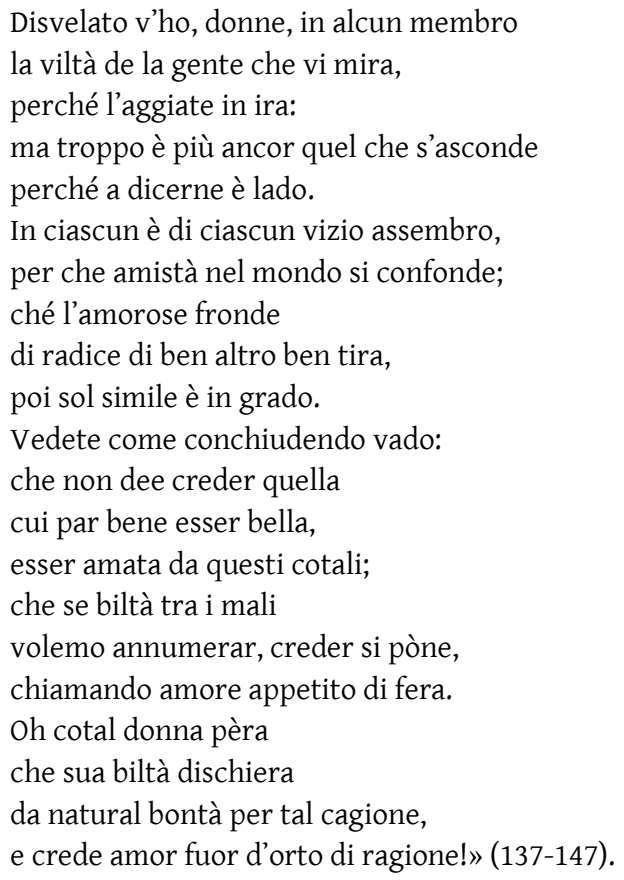

Dunque, in un mondo in cui impera il più orrido dei vizi, l'avarizia, le donne devono nascondere la loro bellezza, devono negarsi a questi uomini che sono «di ciascun vizio assembro», accumulo di ogni possibile turpitudine: l'amore al tempo dell'avarizia, ridotto

anno a presenti, ella n'avrà rossa la tempia, quia destruetur, et luet poenas dignas, non tu, quasi dicat: licet tu pellaris, sicut jam dictum est, tamen melius et honorabilius pertransibis» (Benvenuto da Imola, Comentum, Dartmouth Dante Project, p. 3113). 
a bestiale sensualità («appetito di fera»), deve scomparire, e con esso, si può legittimamente inferire, la procreazione e quindi il genere umano: sembrerebbe quasi di cogliere qui una qualche eco di quelle posizioni gnostico-catare che teorizzavano il rifiuto di procreare come la via più sicura per cancellare l'insostenibile orrore del mondo materiale. Da dove nasce questa desolazione, da cosa è provocata questa repentina e radicale caduta della speranza? Certo, ci sono le motivazioni biografiche: la canzone fu scritta con ogni probabilità fra il 1304 e il 1306, ${ }^{41}$ cioè in uno dei momenti più drammatici della vita di Dante: esule senza speranza di ritorno, solo, povero, braccato da nemici implacabili che lo costringono a un continuo vagabondaggio in cerca di una collocazione sicura che non trova, si può ben capire che il poeta ceda alla disperazione. Ma in verità, nel testo di Doglia mi reca non vi è traccia di autobiografismo: lo sgomento nasce dallo spettacolo della degradazione indotta dall'avarizia, al di fuori e al di là delle vicende politiche contingenti e delle sventure personali. E quindi, ancora una volta, è sul terreno dell'economia politica che dobbiamo inoltrarci per capire le ragioni profonde dello smarrimento del poeta e la sua rinuncia, in questa fase, a ogni ipotesi di riscatto. Il punto è che Dante si è trovato improvvisamente di fronte a un fenomeno in corso di elaborazione già avanzata ma di cui sfuggono, a lui come ai suoi contemporanei, i fondamentali connotati teorici: la nascita del sistema capitalistico. ${ }^{42}$ Non si tratta più,

${ }^{41}$ La datazione è proposta da Umberto Carpi (e accettata fra gli altri da Enrico Fenzi e Claudio Giunta) con ineccepibili argomenti in Carpi, U., La nobiltà di Dante, I, Firenze, Polistampa, 2004, pp. 75-80.

${ }^{42}$ Questo saggio parte dall'assunto che le prime manifestazioni dell'economia capitalista siano riscontrabili nell'Italia del XIII secolo e segnatamente a Firenze. Come si sa, tuttavia, esistono al riguardo due scuole di pensiero: una che conferma la nostra ipotesi, l'altra che vi si oppone. Il dibattito, ormai annoso, è ancora aperto. Riportiamo di seguito, a titolo esemplificativo, due autorevoli testimonianze, rispettivamente a favore dell'una e dell'altra tesi.

«Le fonti di cui disponiamo, per quanto insufficienti, non ci permettono di dubitare del fatto che il capitalismo si affermò fin dal XII secolo. È un dato incontestabile, infatti, che il commercio tra luoghi molto distanti produsse fin da allora ricchezze notevoli. Si è citato più sopra l'esempio di Godrick [cfr. nota 45], che era animato da quello spirito che è proprio dei capitalisti di tutti i tempi, nel pieno significato del termine. Egli ragionava, calcolava; l'unico fine che si proponeva era l'accumulazione dei guadagni. Sono appunto queste le caratteristiche di quel capitalismo, che secondo una scuola di studiosi sarebbe così misterioso, ma che tuttavia si ritrova in ogni epoca, identico nel fondo anche se vario per grado di sviluppo, giacché corrisponde alla naturale tendenza dell'uomo verso la ricchezza. E certo Godrick non era un'eccezione. Il caso, che ci ha tramandato la storia di questo scozzese, avrebbe potuto ugualmente tramandarci quella di un veneziano o di un genovese, e mostrarci così l'uso delle sue stesse facoltà in ambiente assai più favorevole alla loro espressione. L'interesse della figura di Godrick sta nella sua psicologia, che è quella di tutti gli avventurosi mercanti del suo tempo. Egli ci permette di conoscere da vicino il tipo dei nuovi ricchi generati dal commercio, dapprima lungo le coste e poi ovunque, a mano a mano che le attività commerciali penetravano nel continente. Non occorre altro per convincerci dell'importanza acquistata fin da allora dal capitalismo cmmerciale, se si pensa che quelli che conosciamo non sono che gli esemplari di maggior rilievo di una folta schiera», Pirenne, H., Storia economica e sociale del Medioevo, Milano, Garzanti, 1967, pp. 179-180.

«Se si esaminano in blocco i secoli che vanno dall'XI al XVI, delle strane contraddizioni

Revista Española de Filosofía Medieval, 27/1 (2020), ISSN: 1133-0902, pp. 83-120

DOI: 
insomma, della consueta polemica moralistica contro l'avarizia, ma dell'espressione di un atterrito stupore di fronte al mysterium iniquitatis delle prime forme di accumulazione capitalistica e di una nuova funzione del denaro. Scrive con esemplare lucidità Enrico Fenzi:

Il passaggio dall'invettiva contro l'avaro nella terza strofa, alla considerazione allargata che la segue, nella quarta, è dunque essenziale se si vuole cogliere la tensione con la quale Dante articola il passaggio ulteriore, quello che esplode nelle domande rivolte alla Morte e alla Fortuna. Egli ha evitato di incagliare il suo discorso nel solito solco moralistico, non si è limitato ad agitare davanti all'avaro la condanna della sua pazzia e lo spettro della morte che l'attende, e ha invece allargato la sua visuale. Ha colto la dimensione sociale e totalizzante del fenomeno, e a questo punto ha urtato contro qualcosa di essenziale che avverte e che però gli sfugge. ${ }^{43}$

Questo «qualcosa di essenziale» è appunto l'apparizione del sistema capitalistico e delle sue regole, fra le quali c'è quella, per gli uomini del medioevo incomprensibile, della trasformazione del denaro da valore d'uso a valore in sé: nella classica analisi marxiana ${ }^{44}$ il denaro, nell'era del capitale, non serve più ai fini del consumo, con la sua conseguente e continua rimessa in circolazione, ma assume una funzione autonoma che

appaiono immediatamente. Dall'anno 1000 (una data scelta, evidentemente, solo a fini di comodo) e fino al XV secolo i mercanti italiani sono stati incontestabilmente i più forti, i più attivi, i più creativi di tutti i loro colleghi d'Europa: essi hanno avuto delle tecniche, tutta un'attrezzatura materiale e mentale nettamente superiori a quelle dei loro rivali. Ma, da questa superiorità non si può dedurre nessuna modernità reale di questi uomini che non possono essere che del loro tempo: il loro mondo religioso, la loro sfera di sentimenti, la loro idea della vita sono -e non possono non essere- del loro tempo», Romano, R., Tra le due crisi: l'Italia dl Rinascimento, Torino, Einaudi, 1971, p. 96.

Un'ampia discussione sull'argomento si trova inoltre nel volume collettaneo Bolaffi, G. (ed.), La transizione dal feudalesimo al capitalismo, Roma, Savelli, 1974.

${ }^{43}$ Fenzi, E., «Tra etica del dono e accumulazione. Note di lettura alla canzone dantesca Doglia mi reca», in U. Carpi, (ed.), Doglia mi reca ne lo core ardire, Madrid, Asociaciòn Complutense de Dantologia, 2008, pp. 147-195, alla p. 172.

${ }^{44}$ «Nella circolazione M-D-M (Merce-Denaro-Merce) il denaro viene trasformato, alla fine, in merce che serve come valore d'uso. Dunque il denaro è definitivamente speso. Nella forma inversa, D-M-D (Denaro-Merce-Denaro), invece, il compratore spende denaro per incassare denaro come venditore. Alla compera della merce egli getta denaro nella circolazione, per tornare a sottrarnelo a mezzo della vendita della stessa merce. Non lascia andare il denaro che con la perfida intenzione di tornarne in possesso [...] La circolazione semplice delle merci -la vendita per la compera- serve di mezzo per un fine ultimo che sta fuori della sfera della circolazione, cioè per l'appropriazione di valori d'uso, per la soddisfazione di bisogni. Invece, la circolazione del denaro come capitale è fine a se stessa, poiché la valorizzazione del valore esiste soltanto entro tale movimento sempre rinnovato. Quindi il movimento del capitale è senza misura.», Marx, K., Il capitale, Roma, Editori Riuniti, I, 1, pp. 164-168. La «dismisura» dell'accumulazione capitalista qui denunciata da Marx è percepita anche da Dante e descritta con straordinaria lucidità ai vv.70-73: «oh mente cieca che non pò vedere / lo suo folle volere / che 'l numero, ch'ognora a passar bada, / che 'nfinito vaneggia!». 
lo trasforma in «capitale», innescando una spirale di «valorizzazione del valore» che tende all'infinito e blocca la redistribuzione. ${ }^{45}$ Ecco quindi l'esasperato appello di Dante alla Morte e alla Fortuna perché diano conto della loro incapacità di rompere il cerchio dell'accumulazione capitalistica: «Morte che fai? che fai, fera Fortuna, / che non solvete quel che non si spende? / se 'l fate, a cui si rende?» (90-92). La risposta a queste domande, seicento anni prima di Marx, non può essere che una dichiarazione di impotenza a capire: «Non so, poscia che tal cerchio ne cinge / che di là su ne riga» (9394). In questo smarrito «Non so» si concentra tutta la drammaticità dell'impasse in cui si trova il poeta di fronte a un fenomeno di cui avverte la terribile grandiosità ma che non trova spiegazione nel suo sistema di valori e di conoscenze..$^{46}$ Ancora una volta giova ripetere che, se valesse l'ipotesi di un Dante conservatore reazionario, sarebbe logico che egli si fermasse qui, alla denuncia della catastrofe rinunciando a ogni intervento che non sia quello del ripristino del «buon tempo antico». Ebbene, non è così: proprio a partire dal «cuore di tenebra» della canzone, nel momento in cui l'angoscia del presente si fa quasi insostenibile («Qui si raddoppia l'onta, / se ben si guarda là dov'io addito», 99-100) ci troviamo improvvisamente di fronte a una via di scampo: descrivendo come non si deve donare, Dante propone per contrasto un'etica del dono su cui costruire un'alternativa al ferreo dominio dell'avarizia. Ecco i versi (118126): «I' vo' che ciascun m'oda: / chi con tardare, e chi con vana vista, / chi con sembianza trista / volge il donare in vender tanto caro, / quanto sa sol chi tal compera paga. / Volete udir se piaga? / Tanto chi prende smaga, / che 'l negar poscia non li pare amaro. / Così altrui e sé concia l'avaro».

Dunque, un mondo in cui la pratica del dono disinteressato, pronto e lieto

${ }^{45}$ Il fatto che l'analisi marxiana sia applicabile all'economia del medioevo è confermato da una ricchissima tradizione documentale. Citiamo qui come caso esemplare un passo della biografia di Godrick di Finchal, mercante scozzese del XII secolo, in cui è perfettamente descritto il processo di accumulazione capitalista nei termini definiti da Marx: «Unde et mercandi gratia frequenter in Daciam ibat et aliquoties in Flandriam navigii remige pervolabat; et dum oportunitas juvabat, littora marina circumiens, multoties ad Scotorum fines deveniebat. In quibus singulis terrarum finibus aliqua rara et ideo pretiosiora reperiens, ad alias secum regiones transtulit, in quibus ea maxime ignota fuisse persensit, quae apud indigenas desiderabiliora super aurum existiterant; et ideo pro his quaeque alia, aliis terrarum incolis concupiscibilia, libentius et studiosissime commutando comparabat. De quibus singulis negotiando plurimum profecerat er maximas opum divitias in sudore vultus sui sibi perquisierat, quia hic multum venumdabat quod abili ex parvi pretii sumptibus congregaverat», Reginaldo Monaco, Libellus de vita et miraculis S. Godricii, London, Surtees Society, 1847, p.29.

${ }^{46}$ «Per Dante (e per gli uomini del suo tempo), quali che fossero le loro pratiche economiche, non era possibile uscire dallo schema che voleva il denaro o utilizzato in vista del consumo, o immobilizzato quale tesoro. Poi, definisce la dimensione infinita, «senza misura» della valorizzazione capitalistica «ch'ognora a passar bada» il «numero» sempre provvisorio, limitato e mobile in cui quella dimensione s'incarna: il che alle categorie morali del tempo appariva come una vera e propria bestemmia, un parto diabolico assimilabile sempre e comunque all'usura pura e semplice», Fenzi, Tra etica del dono e accumulazione. Note di lettura alla canzone dantesca Doglia mi reca, op. cit., p. 177. 
sostituisca la malavoglia e la compiacenza vanitosa con cui dona l'avaro, può restituire ai rapporti sociali quella nobiltà cancellata dal trionfo dell'avarizia. Ma naturalmente i versi citati di Doglia mi reca non costituiscono un unicum: un evidentissimo riscontro di quanto il tema della liberalità e del dono si imponesse in quegli anni alla sensibilità di Dante come antidoto al veleno dell'avarizia si ha per esempio nel Convivio, là dove la scelta del volgare è spiegata come un esempio di «pronta liberalitade». ${ }^{47} \mathrm{E}$ del resto non ci sarà bisogno di sottolineare come le vicende dell'esilio avessero messo il poeta di fronte alla necessità di ricercare, per la propria sopravvivenza, il sostegno dei potenti presso i quali di volta in volta trovava rifugio, sostegno che spesso era elargito in forme lontane dall'ideale della «pronta liberalitade» («Tu proverai sì come sa di sale / lo pane altrui e come è duro calle / lo scendere e 'l salir per l'altrui scale», Par. XVII, 58-60).

L'etica del dono, naturalmente, non è un'invenzione dantesca: essa fa parte integrante della civiltà cortese come dimostrano innumerevoli testimonianze storiche e letterarie. Ma sarà utile ricordare che, se per Dante il connotato cortese era senza dubbio il punto di riferimento fondamentale, l'etica del dono ha confini spaziali e temporali ben più ampi: come infatti insegna l'antropologia a partire dagli studi di Marcel Mauss, la società fondata sul profitto e sull'interesse è una creazione abbastanza recente che ha sostituito solo parzialmente quella guidata dai princìpi del dono e del disinteresse, dominante in tutto il mondo della tradizione e tuttora vigente nelle società che conservano tratti arcaici ${ }^{48}$ Nessuno pensa, ovviamente, di istituire avventurosi

47 «Quando è mostrato per le sufficienti ragioni come, per cessare disconvenevoli disordinamenti, converrebbe a le nominate canzoni aprire e mostrare comento volgare e non latino, mostrare intendo come ancora pronta liberalitade mi fece questo eleggere e l'altro lasciare. Puotesi adunque la printa liberalitade in tra cose notare, le quali seguitano questo volgare, e lo latino non averebbero seguitato. La prima è dare a molti; la seconda è dare utili cose; la terza è, sanza essere domandato lo dono, dare quello [...] Primamente, però che la vertù deee essere lieta, e non trista in alcuna sua operazione, onde se 'l dono non è lieto nel dare e nel ricevere, non è in esso perfetta vertù, non è pronta», Conv. I, 8. Ma sul tema del dono il Convivio si sofferma anche in numerosi altri luoghi (I, 3; IV, 12; IV, 27).

${ }^{48}$ «Sottoponiamo ora alla prova decisiva l'altra nozione da noi contrapposta a quella di dono e di disinteresse: la nozione di interesse, di ricerca individuale dell'utile. Anche questa nozione opera diversamente rispetto al modo in cui agisce nel nostro spirito. Se un qualche motivo equivalente anima capi trobriandriani o americani, clan andamani, ecc., o animava, un tempo, generosi Indiani, nobili Germani o Celti nel fare doni o spese, non si tratta ora né si trattava prima di un motivo legato alla fredda ragione del mercante, del banchiere e del capitalista. Presso queste civiltà, la molla dell'interesse funziona diversamente che da noi. Si tesaurizza, ma allo scopo di spendere, di «obbligare», di disporre di «uomini ligi«. Si effettuano scambi, ma di oggetti di lusso, di ornamenti, di vestiario, o di cose che vengono immediatamente consumate, di banchetti. Si ricambia ad usura, ma per umiliare colui che ha donato o scambiato per primo, non soltanto per ricompensarlo della perdita che gli procura un «consumo differito». Esiste un interesse, ma questo interesse è solo analogo a quello che, a quanto si dice, ci guida. Tra l'economia relativa amorfa e disinteressata, all'interno dei sottogruppi, che regola la vita dei clan australiani o americani del Nord (Est e Prateria), da un lato; e l'economia individuale basata sul puro interesse, che le nostre società hanno conosciuto, almeno parzialmente, subito dopo la sua scoperta da parte delle 
collegamenti fra la speculazione dantesca sulla «pronta liberalitade» e i risultati dei moderni studi antropologici; basterà, per marcare la centralità del tema, osservare come esso fosse largamente presente nella riflessione dei contemporanei di Dante; l'etica del dono era insomma uno dei capisaldi della pedagogia cortese con la quale la borghesia mercantile tentava di riqualificarsi: abbiamo già visto in proposito Brunetto, ma fra le innumerevoli ulteriori testimonianze ci sembra particolarmente significativa quella di un testo come il Novellino, in cui l'etica del dono si propone fin dall'esergo ("Questo libro tratta [...] di belle valentie e doni») per culminare nella esemplare novella LXXXIX, Della gran cortesia de' gentili uomini di Brettinoro..$^{49} \mathrm{E}$ non sarà forse un caso che proprio Bertinoro venga citata da Dante fra gli esempi di decadenza dopo un'epoca di splendore cortese ( $« 0$ Bretinoro, ché non fuggi via, / poi che gita se n’è la tua famiglia / e molta gente per non esser ria?», Purg. XIV, 112-114)..$^{50}$ Ma l'etica del dono affonda le sue radici ben al di là della cultura cortese, trovando riscontro in numerosi luoghi scritturali, fra i quali spicca il celebre «mutuum date nihil inde sperantes» $(L c, 6,35)$ che sarà riferimento essenziale per tutte le correnti pauperistiche e bandiera di tutte le polemiche contro l'asservimento della Chiesa alle ragioni del denaro e del profitto. Tuttavia si sbaglierebbe se si interpretasse l'etica del dono in un senso puramente ascetico, come spossessamento volontario dei beni materiali per conquistare quella perfezione morale che solo la povertà può dare: in realtà il dono si inserisce in un progetto di società dominata dalla charitas che non esclude l'uso dei beni purché esso avvenga all'interno di un reciproco e volontario rapporto di solidarietà fra $\mathrm{i}$ componenti della comunità e sia regolato da una corretta conoscenza della natura dei beni stessi e da una attenta distinzione fra necessario e superfluo. ${ }^{51}$ Del resto, la

popolazioni semitiche e greche, dall'altro; tra questi due tipi di economia si è scaglionata, io dico, tutta una immensa serie di istituzioni e di avvenimenti economici, non certo guidata dal razionalismo economico, di cui si costruisce così volentieri la teoria. Lo stesso termine interesse è recente, di origine tecnico-contabile: interest, latino, che si usava scrivere nei libri di conti, di fronte alle rendite da percepire. Nelle morali antiche più epicuree, non l'utilità materiale, ma il bene e il piacere vengono ricercati. È stata necessaria la vittoria del razionalismo e del mercantilismo perché fossero poste in vigore ed elevate all'altezza di principi, le nozioni di profitto e di individuo», Mauss, M., Saggio sul dono. Forma e motivo dello scambio nelle società arcaiche, in Teoria generale della magia e altri saggi, Torino, Einaudi, 1965, pp. 153-292, alle pp. 282-283.

${ }^{49}$ "Intra gli altri bei costumi de' nobili di Brettinoro, era il convivare e che non voleano, che uomo vendereccio vi tenesse ostello. Ma una colonna di pietra era nel mezzo del castello, alla quale, come entrava dentro il forestiere, era menato; ed a una delle campanelle, che ivi erano, conveniali mettere le redine del cavallo, o arme, o cappello che avesse. E come la sorte gli dava, così era menato alla casa, per lo gentile uomo al quale era attribuita quella campanella, ed onorato secondo suo grado. La qual colonna e campanella furon trovate, per tollere matera di scandalo intra li detti gentili; ché ciascuno prima correva a menarsi a casa li forestieri, sì come oggi quasi si fugge», Il Novellino, Milano, Rizzoli, 1975, p. 137.

${ }^{50}$ Bertinoro, cittadina romagnola tra Forlì e Cesena, fu celebre nel XII secolo per la liberalità della famiglia dei Mainardi che ne ebbe la signoria e che si estinse nel 1177. Ad essa allude probabilmente il v. 113.

${ }^{51}$ «'Usare' (uti) attraverso la lente chiarificatrice della 'povertà', ossia della verifica dei bisogni 
testualità che riguarda la funzione civile del dono legata alla misura e al rapporto equilibrato fra donatore e beneficiato è ricchissima anche all'esterno del contesto religioso, costituendo, ad esempio, un topos della letteratura trobadorica (si veda Sordello ${ }^{52}$.

Il testo archetipo all'origine di questo modello resta comunque quel passo degli Atti degli Apostoli che reca:

Multitudinis autem credentium erat cor unum et anima una, nec quisquam eorum, quae possidebat, aliquid suum esse dicebat, sed erant illa omnia communia. Et virtute magna reddebant apostoli testimonium resurrectionis Iesu Christi Domini nostri; et gratia magna erat in omnibus illis. Neque enim quisquam egens erat inter illos. Quotquot enim possessores agrorum aut domorum erant vendentes afferebant pretia eorum, quae vendebant et ponebant ante pedes apostolorum. ${ }^{53}$

È lo stesso insegnamento che troviamo in Paolo, espresso in forma più icastica, quando l'apostolo esorta i suoi interlocutori a comportarsi come coloro che pur «possedendo» tutto non «hanno» nulla («nihil habentes et omnia possidentes»" ${ }^{54}$ ). Insomma, come nota Giacomo Todeschini, ${ }^{55}$ il dono entra a far parte di un'ipotesi di economia politica fondata su una distribuzione e una gestione dei beni mirate non al possesso e all'arricchimento personale, ma al bene comune e al recupero di coloro che

effettivi e delle necessità specifiche, appare categorialmente rivelatore dell'identità dei cristiani», Todeschini, G., I mercanti e il tempio. La società cristiana e il circolo virtuoso della ricchezza fra Medioevo ed Età Moderna, Bologna, Il Mulino, 2002, p. 125.

${ }_{52}$ «Quar per larguesa amesurada / anc nulz om larcs non pres baisada, / mas per largueza franca e folla / destrui 'l seu e son pretz afolla; / e qui destrui lo seu e pert / son pretz, non a bon sen ni cert. / Per qu'om deu, qui tot vol salvar, / per la mediana via anar», Sordello, Aissi col tesaur, in Köhler, Sociologia della fin'amor, op. cit., pp. 78-79.

${ }^{53}$ Act. IV, 32.

${ }^{54} 2$ Cor. VI, 10.

${ }^{55}$ «ll dono viene sempre più chiaramente inteso come un comportamento economico in grado di reintegrare nel consorzio civile chi ne sia uscito per ragioni economiche o fisiche, come un comportamento economico in grado di restaurare la solvibilità di soggetti privati (ma anche pubblici), e nello stesso tempo come una strategia dello scambio in grado di generare obbligazioni utilmente spendibili a favore della Salvezza propria o altrui: ma proprio per questo come un momento da considerare con attenzione e cautela. In effetti «donare» ovvero elargire o dare in elemosina, assume in questa luce tutto il rilievo di un movimento economico, che dall'interno del patrimonio familiare (della familia costituita da consanguinei, ma anche dalla familia monastica o da quella ecclesiale: i domesticos fidei del de officiis di Ambrogio) raggiunge per gradi i margini o l'esterno della comunità, della città, della dimensione pubblica, ed in se stesso è capace di qualificare come cristiano, come accettabile m oralmente ed economicamente, qualora lo risolva, ogni singolo momento di disagio, di perdita, di impoverimento. [...] «Donare», in un'economia mercantile come quella delle città due e trecentesche, viene codificato fra i comportamenti economici come il momento decisivo, come la transazione e il rapporto di scambio essenziale, non solo per ricostituire quanto si sia smarrito nella corsa della comunità cristiana alla ricchezza, ma anche per verificare quanto di quello che è diventato marginale valga la pena di recuperare», Todeschini, I mercanti e il tempio, op. cit., pp. 208-209. 
per qualche ragione si sono trovati esclusi o marginali ( $i$ «poveri»). In altre parole, il dono non si configura come pura prassi caritatevole o testimonianza virtuosa, ma si rivela una componente essenziale dell'interpretazione cristiana dell'economia politica, intesa a costruire la comunità di coloro che saranno salvati, la congregatio fidelium di Tommaso. ${ }^{56}$ Ma tra la fine del XIII e i primi anni del XIV secolo (come testimonia Doglia mi reca) l'etica del dono, pur continuando a restare centrale nell'elaborazione del pensiero cristiano appare ormai annichilita nella prassi sociale: Dante lo poteva constatare anche solo considerando la variazione del lessico nell'uso scrittorio dei documenti bancari, in cui nel corso del Duecento, come documenta Arrigo Castellani, la voce «dono» aveva assunto il significato di «interesse», e "donare» quello di «corrispondere un interesse». ${ }^{57}$

Ora, è appunto questo perdersi del valore del dono, sostituito come struttura portante del sistema economico dall'accumulazione capitalista, che è percepito da Dante come la vera radice dell'avarizia; $\mathrm{e}$ il suo ripristino sarà dunque condizione essenziale per avviare la costruzione di una società nuova fondata non più sull'utile individuale ma sull'uso collettivo delle risorse che sono «possedute» da tutti ma che nessuno «ha» come proprie (nihil habentes et omnia possidentes). L'episodio di Provenzano Salvani (Purg. XI, 121-138) ce ne offre una chiara esemplificazione: Provenzano sconta in Purgatorio il peccato di superbia, ma questa superbia sembra in realtà essere molto vicina all'avarizia, ${ }^{58} \mathrm{o}$ meglio, essere una sua variante, uno dei molti modi in cui l'avarizia può manifestarsi. E infatti la descrizione che Oderisi fa di Provenzano è centrata, più che sulla superbia, sull'avidità di potere e di dominio: «-Quelli è-, rispuose, -Provenzan Salvani; / ed è qui perché fu presuntüoso / a recar Siena tutta a le sue mani». L'immagine di Provenzano che allunga le mani su Siena nella bramosia di possederla tutta ci porta, più che nel campo semantico della superbia, in quello appunto dell'avidità e della dismisura, così come, simmetricamente, il riscatto dal peccato avviene grazie a un atto riparatore che non è tanto fondato sul rapporto superbia vs. umiltà, quanto su quello avarizia vs. dono: «-Quando vivea più glorioso-, disse, / -liberamente nel campo di Siena, / ogne vergogna diposta, s'affisse; / e lì, per trar l'amico suo di pena, / ch'e' sostenea ne la prigion di Carlo, / si condusse a tremar per ogne vena-».

L'assimilazione dell'avarizia con la brama di potere e con la superbia è del resto testimoniata da numerosi altri luoghi della Commedia: per esempio, dalle parole con cui

${ }^{56}$ «Il dono [...] era funzionale a una concezione della marginalità sociale ed economica come perdita da recuperare: si trattava dunque di una carità dai molteplici significati produttivi. Essa avrebbe dovuto far sì che $\mathrm{i}$ «poveri« divenissero membri utili della società, imparando un mestiere, guarendo da ogni sorta di mali fisici e spirituali, convertendosi, in sostanza, a una Cristianità effettiva, sia latamente religiosa e sociale che specificamente economica», ibid., p. 190.

${ }^{57}$ Castellani, A., Nuovi testi fiorentini del Dugento, Firenze, Sansoni, 1952, II, p. 865.

${ }^{58}$ Sulla coincidenza fra avarizia e superbia la tradizione teologica duecentesca si era già espressa con la massima chiarezza. Si veda per esempio in San Tommaso: «avaritia est non solum pecuniae, sed etiam scientiae et altitudinis, cum supra modo sublimitas ambitur», Summa Theologiae II, 2, q. CXVIII, art. 1. 
Adriano V definisce il suo peccato in Purg. XIX, 106-111: «La mia conversìone, oimè!, fu tarda; / ma, come fatto fui roman pastore, / così scopersi la vita bugiarda. / Vidi che lì non s'acquetava il core, / né più salir potiesi in quella vita; / per che di questa in me s'accese amore». Il potere, dunque, sembra confliggere fatalmente con la charitas che sola può giustificare e legittimare l'architettura di un'economia fondata sullo scambio e sul profitto, altrimenti condannata ad assumere una fisionomia usuraria. Solo la gratuità del dono può evitare questo esito: e difatti, sempre nel Purgatorio (XXII, 67-69) assumono il valore di un sigillo definitivo di salvezza le parole che Stazio rivolge a Virgilio: «Facesti come quei che va di notte, / che porta il lume dietro e sé non giova, / ma dopo sé fa le persone dotte», epitome di una prassi sociale ispirata ai tre princìpi che fino dalla testualità patristica avevano fondato l'economia politica della società cristiana: charitas, amicitia e fidelitas. La charitas, intesa come senso di solidarietà e legame virtuoso da cui nasce l'utilità generale, è il valore che fonda ogni iniziativa economica della comunità cristiana (sempre però sottoposta a un'attenta valutazione razionale in modo da non trasformarsi in sperpero ingiustificato o inopportuno ${ }^{59}$ ); l'amicitia è il sentimento che si stabilisce fra il donatore e il destinatario del dono e che, per esempio, nella meccanica del prestito va a sostituire, sotto forma di 'riconoscenza', l'obbligo dell'interesse legale; la fidelitas, infine, è l'appartenenza alla comune professione di fede cristiana che dà il diritto di usare i beni del mondo da cui gli infedeli sono esclusi. ${ }^{60}$ Dall'intreccio e dalla cooperazione di charitas, amicitia e fidelitas nasce il «bene comune» alla cui compiuta realizzazione tende l'economia politica cristiana. Dante ebbe chiarissima l'idea del bene comune come forma definitiva della sua città ideale: su questo versante fu indubbiamente centrale per lui (come dimostra Umberto Carpi $^{61}$ ) la suggestione di Remigio dei Girolami, che fra il 1270 e il 1310 fu lettore nello Studio fiorentino di Santa Maria Novella e che certo fu tra quei «religiosi» e quei «filosofanti» da cui il poeta ci dice di avere appreso i primi insegnamenti filosofici. ${ }^{62} \mathrm{Il}$ bene comune, così come è rappresentato da Remigio in un celebre passo del suo De bono

${ }^{59}$ ««Donare» [...] appare nel discorso economico duecentesco come atto codificato strettamente connesso al criterio prudente di scelta: chi dona deve saper scegliere, come il «cambiavalute« fra moneta falsa e vera, fra bisogni autentici, cioè determinati da effettive miserie, e bisogni rivelatori di colpevole oziosità o di molteplici dissipazioni. L'utilità, il commodum derivante quale controdono dalla riconoscenza del beneficato, dipendono interamente dalla dignità morale di chi riceve il dono. Il donatore non appare, in questa luce, un casuale benefattore del prossimo, ma piuttosto un uomo d'affari che, avendo a cuore la propria famiglia e la propria comunità, sa scoprire, distinguendo nel magma confuso dei comportamenti e delle intenzioni dei «poveri», quali siano le situazioni di disagio (di perdita, di smarrimento, di decadimento, di abbandono) da alleviare secondo i principi di una razionalità funzionale all'utile spirituale-politico della collettività «fedele», Todeschini, I mercanti..., pp. 208-209.

${ }^{60}$ Chiarissimo in proposito Graziano: «Fidelis hominis totus mundus divitiarum est, infidelis autem nec obolus», Decretum, XIV, $4,11$.

${ }^{61}$ Cfr. Carpi, La nobiltà di Dante, op. cit., p. 56.

${ }^{62}$ «E da questo imaginare cominciai ad andare là dov'ella [la filosofia] si dimostrava veracemente, cioè ne le scuole de li religiosi e a le disputazioni de li filosofanti», Conv. II, 12. 
comuni, ${ }^{63}$ richiama irresistibilmente alla memoria l'esempio di collaborazione armoniosa e solidale che ci viene offerto da Dante con la descrizione dell'Arsenale dei Veneziani in Inf. XXI, 11-15, descrizione che certo può sembrare incoerente se messa in rapporto con l'avido egoismo dei barattieri a cui fa da introduzione, ma che acquista un senso appunto proprio come icastica contrapposizione del bene comune al bene grettamente individuale: «[...] in quella vece / chi fa suo legno novo e chi ristoppa / le coste a quel che più vìaggi fece; / chi ribatte da proda e chi da poppa; / altri fa remi e altri volge sarte; / chi terzeruolo e artimon rintoppa».

Un altro caso esemplare di dedizione al bene comune lo abbiamo con Pier Pettinaio, ricordato da Sapia in Purg. XIII, 127-129 («[...] a memoria m'ebbe / Pier Pettinaio in sue sante orazioni, / a cui di me per caritate increbbe»), a proposito del quale, con un chiaro riferimento alla teoria del bene comune tradotta nella quotidianità, annota l'Anonimo Fiorentino:

Piero Pettinagno fece in Camollia di Siena una bottega di pettini, et elli fu cittadino sanese, et dicesi ch'egli andava a Pisa a comperare i pettini, et comperavagli a dozzina; poi che gli avea comperati, egli se ne venia con questi pettini in sul ponte vecchio di Pisa, et sceglieva i pettini, et se niuno ve ne avea che fosse fesso o non buono egli il gettava in Arno. Fugli detto più volte perché il pettine sia fesso o non così buono, egli pur vale qualche denaro, vendilo per fesso: Piero rispondea: Io non voglio che niuna persona abbia da me mala mercatanzia. ${ }^{64}$

Ancora, al principio del bene comune è riconducibile la leggenda di Traiano riportata in Purg. X, 73-93:

La miserella intra tutti costoro

pareva dir «Segnor, fammi vendetta

di mio figliuol ch'è morto, ond'io m'accoro»;

ed elli a lei rispondere: «Or aspetta

tanto ch'i' torni»; e quella: «Segnor mio»,

come persona in cui dolor s'affretta,

«se tu non torni?»; ed ei: «Chi fia dov'io,

la ti farà»; ed ella: «L'altrui bene

a te che fia, se 'l tuo metti in oblio?»;

ond'elli: «Or ti conforta; ch'ei convene

${ }^{63}$ «Quod etiam apparet ex hoc quia homines naturaliter congregantur et faciunt civitatem vel aliud comune propter utilitatem propriam ad subveniendum defectibus vite humane quibus unus subvenire non potest, ut alter suppleat ubi alter deficit, quia unus facit calceamenta, alius domos, alius vestimenta, alius colit terram, alius facit arma, et sic de ceteris quibus indiget humana vita», Remigio dei Girolami, De bono comuni, Firenze, Nerbini, 2014, p. 186.

${ }^{64}$ Anonimo Fiorentino, Commento, Dartmouth Dante Project, p. 1649. 
ch'i' solva il mio dovere anzi ch'i' mova:

giustizia vole e pietà mi ritene».

Così come «giustizia» e «pietà» muovono Traiano, il «ben fare» muove Romeo di Villanova a operare per il bene comune (Par.VI, 127-142) nel più assoluto disinteresse per il vantaggio personale. Insomma, da questi come da tanti altri luoghi della Commedia, sembra di poter concludere che la riflessione dantesca va ben al di là dello sterile rimpianto di maniera, tipico di ogni conservatore-reazionario, per un passato tanto idealizzato quanto irrecuperabile: Dante dimostra, al contrario, di avere perfettamente percepito il definitivo tramonto del mondo feudale e della civiltà cortese, e di avvertire con drammatica urgenza la necessità di una svolta radicale che possa gettare le basi di un mondo diverso. ${ }^{65}$ I materiali teorici e dottrinali per avviare questo percorso, del resto, non mancavano: come abbiamo visto fin qui, depositi immensi di alternative alla mercificazione verso cui stava precipitando l'occidente tardomedievale si trovavano nella millenaria tradizione cristiana a partire dallla testualità scritturale per giungere fino alle contemporanee investigazioni di un Olivi o di un Remigio dei Girolami, passando attraverso gli elementi di economia politica affrontati dalla Scolastica, in primis, naturalmente, da Tommaso d'Aquino; e non occorrerà ribadire ancora una volta il contributo essenziale della tradizione aristotelica.

Dunque, se all'altezza di Doglia mi reca, nel momento più angoscioso della sua vicenda politica e umana, sembra prevalere in Dante una sorta di resa alla disperazione, questa deriva nichilistica non è affatto definitiva, anzi, convive con una apertura progettuale verso una possibile alternativa che via via si fa sempre più energica fino a culminare nella Commedia e, sul piano della speculazione teorica, nel De Monarchia. Ma già nel Convivio, la cui elaborazione è contemporanea alla stesura di Doglia mi reca, la cruciale riflessione sulla nobiltà posta nel IV Trattato a commento della canzone Le dolci rime prefigura un modello di società estraneo a quello fondato sull'accumulazione delle ricchezze, sviluppando una critica radicale della nozione stessa di ricchezza:

Come detto è, la imperfezione de le ricchezze non solamente nel loro avvenimento si può comprendere, ma eziandio nel pericoloso loro accrescimento; e però che in ciò più si può vedere di loro difetto solo di questo fa menzione lo testo, dicendo quelle,

\footnotetext{
${ }^{65}$ «A questa nuova realtà economica, nella quale il conflitto di interessi fra le corporazioni sostituisce la solidarietà fra le classi, Dante contrappone non certo il rimpianto di mitici costumi feudali del passato, ma bensì l'esigenza di modernissimi princìpi statuali che ai diversi livelli (nazionale o sovranazionale) siano capaci di mediare ra le parti in conflitto, disattivando la causa dei conflitti stessi, cioè l'avarizia: sedatis fluctibus blande cupiditatis. Dante ha perfettamente chiari sia la nuova realtà economica che mette in crisi l'ordine feudale, sia gli antidoti istituzionali che potrebbero incanalarne «virtuosamente» le energie distruttive, reintroducendo il senso del bene comune (ossia la percezione della differenza de le cose in quanto sono ad alcuno fine ordinate), che non può essere avvertito dalla prospettiva dei gruppi comunali, le famigerate "Arti», che hanno frammentato corporativamente il popolo fiorentino», Pinto, R., Le donne innamorate come soggetto politico, in Carpi (ed.), Doglia mi reca ne lo core ardire, op. cit., pp. 97-146, alla p. 120.
} 
quantunque collette, non solamente non quietare, ma dare più sete e rendere altri più defettivo e insufficiente [...] E per questo modo le ricchezze pericolosamente nel loro accrescimento sono imperfette, che, sommettendo ciò che promettono, apportano lo contrario. Promettono le false traditrici sempre, in certo numero adunate, rendere lo raunatore pieno d'ogni appagamento; e con questa promissione conducono l'umana volontade in vizio d'avarizia [...] Promettono le false traditrici, se bene si guarda, di torre ogni sete e ogni mancanza, e apportare ogni saziamento e bastanza; e questo fanno nel principio a ciascuno uomo, questa promissione in certa quantità di loro accrescimento affermando: e poi che quivi sono adunate, in loco di saziamento e di refrigerio danno e recano sete di casso febricante intollerabile; e in loco di bastanza recano nuovo termine, cioè maggiore quantitade e desiderio, e, con questa, paura grande e sollicitudine sopra l'acquisto. Sì che veramente non quietano, ma più danno cura, la qual prima sanza loro non si avea. ${ }^{66}$

Dunque la dismisura che spinge a un accumulo di ricchezze senza fine, non solo è all'origine di un ordine sociale iniquo e fondamentalmente malvagio (si ricordi nella Commedia, Inf. XVI, 73-74, il grido di Dante: «La gente nuova e i sùbiti guadagni / orgoglio e dismisura han generata»), ma è anche fonte di infelicità per chi ne è vittima, secondo una consolidata tradizione che risale alla nozione aristotelica della medietas come cardine del comportamento virtuoso in opposizione alla dismisura. ${ }^{67}$

Tuttavia, una volta contestata così radicalmente una società fondata sulla mercificazione, restava aperta la necessità di procedere a una pars construens che non poteva esaurirsi nell'invettiva contro le «divizie» né appagarsi di un'etica del dono che avrebbe fatalmente trasformato la tensione verso il nuovo nel tentativo di un recupero dei valori feudali-cortesi che a questa altezza Dante avverte come definitivamente tramontati. Occorreva dunque uscire dall'alternativa nichilismo/ conservatorismo per avventurarsi in territori inesplorati, e il primo passo in questa direzione è appunto il rifiuto di ogni forma di mercificazione, condizione preliminare per entrare nella dimensione del «bene comune»: addirittura, nel Convivio il rifiuto della mercificazione si spinge fino a negare lo status di «litterati» a quegli intellettuali che fanno commercio del loro sapere. ${ }^{68}$ Qui torna naturalmente centrale il valore del dono, ma, come si è detto, perché esso diventi sistema è necessario che non resti un puro imperativo etico

${ }^{66}$ Conv. IV, 12.

${ }^{67}$ «Medietas autem duarum maliciarum, huius quidem secundum abundaciam, huius autem secundum defectum, et adhuc huic, has quidem deficere, has autem superabundare ab eo quod oportet et in passionibus et in operacionibus, virtutem autem medium et invenire et eligere. Propter quod secundum substanciam quidem et racionem quid est esse dicentem, medietas est virtus, secundum optimum autem et bene, extremitas», Aristotele, Ethica Nicomachea, II, 6, in Aristoteles Latinus, XXVI, Leiden, ed. R.A. Gauthier, 1971, p. 171.

${ }^{68}$ «E a vituperio di loro dico che non si deono chiamare litterati, però che non acquistano la lettera per lo suo uso, ma in quanto per quella guadagnano denari o dignitate; sì come non si dee chiamare citarista chi tiene la cetera in casa per prestarla per prezzo, e non per usarla per sonare», Conv. I, 9. 
o, peggio ancora, l'ornamento morale che giustifica il dominio sociale: il dono deve diventare prassi collettiva, fondamento di una nuova economia politica da cui sia bandito una volta per tutte ogni elemento di profitto soggettivo e di interesse personale. La bramosia del possesso e dell'affermazione di sé, il calcolo del dare e dell'avere, la riduzione dell'esistenza al computo utilitaristico di costi e benefici dovrà essere sostituita dalla disponibilità a donare e a ricevere senza secondi fini, restandone appagati in spirito di carità. Una lettura in questa chiave della Commedia, e in particolare del Paradiso, offre risultati assolutamente probanti, a cominciare dalla risposta che Piccarda dà alla domanda di Dante se gli spiriti che si trovano nel cielo della Luna non preferirebbero trovarsi in «più alto loco». Piccarda risolve la questione così (Par.III, 7072): «Frate, la nostra volontà quî̀eta / virtù di carità, che fa volerne / sol quel ch'avemo, e d'altro non ci asseta».

E non molto più avanti sarà Giustiniano, con una evidente condanna dell'ambizione mirata al successo (variante in chiave "civile» dell'interesse utilitaristico), a ribadire che il desiderio di onore e fama allontana da Dio l'uomo che invece dovrebbe appagarsi di un equo riconoscimento legato ai meriti (Par.VI, 112-120): "Questa piccola stella si correda / d'i buoni spirti che son stati attivi / perché onore e fama li succeda: / e quando li disiri poggian quivi, / sì disvìando, pur convien che i raggi / del vero amore in su poggin men vivi. / Ma nel commensurar d'i nostri gaggi / col merto è parte di nostra letizia, / perché non li vedem minor né maggi». E non è certo casuale che questa esposizione di Giustiniano introduca lo spirito di Romeo di Villanova, esempio preclaro di generosità al di fuori e al di sopra di ogni calcolo interessato.

Dunque il destino dell'uomo su questa terra può essere felice solo a patto di abbandonare ambizioni e ossessioni di potere e di possesso, accettando invece con libera e serena coscienza di percorrere la strada che ci è stata assegnata secondo natura. Dice Carlo Martello (Par.VIII, 139-148): "E se 'l mondo laggiù ponesse mente / al fondamento che natura pone, / seguendo lui, avria buona la gente. / Ma voi torcete a la religione / tal che fia nato a cignersi la spada, / e fate re di tal ch'è da sermone; / onde la traccia vostra è fuor di strada». Seguire dunque la natura, condividere fraternamente il destino nostro e quello degli altri, appagarsi di un ordine armonioso voluto da una volontà superiore: le lotte e gli odi che ci dilaniano in questa «aiuola che ci fa tanto feroci» nascono appunto dalla trasgressione di quest'ordine, stravolto dalla cupidigia. E sarà Beatrice, quasi alla fine del poema, a pronunciare la più lucida diagnosi del male che affligge l'umanità: «Oh cupidigia che i mortali affonde / sì sotto te, che nessuno ha podere / di trarre li occhi fuor de le tue onde! / Ben fiorisce nelli uomini il volere; / ma la pioggia continüa converte / in bozzacchioni le susine vere». ${ }^{69}$

Questa desolata constatazione del naufragio dei «mortali», resa ancora più drammatica dal ricorso a un registro plebeo («bozzacchioni», «susine»), viene però

${ }^{69}$ Par. XXVII, 121-126 
subito smentita dalla profezia che chiude il Canto con una vigorosa apertura su un prossimo riscatto: «Ma prima che gennaio tutto si sverni / per la centesma ch'è là più negletta, / raggeran sì questi cerchi superni, / che la fortuna che tanto s'aspetta, / le poppe volgerà u' son le prore, / sì che la classe correrà diretta; / e vero frutto verrà dopo 'l fiore». Il passo è cruciale: Dante crede ancora, dopo la catastrofe di Arrigo VII (il Canto è infatti sicuramente posteriore al 1313, data della morte di Arrigo), alla ineluttabilità di una palingenesi, anzi, alla sua manifestazione in tempi rapidi (la elaborata perifrasi astronomica dei vv. 142-143 va intesa come una litote che proclama l'imminenza del cambiamento ${ }^{70}$ ). E tuttavia, nonostante la violenza con cui, con accenti evidentemente millenaristi, Dante denuncia la disgregazione di una civiltà e il prossimo avvento di un mondo rigenerato, il giudizio che ha coinvolto molta parte del dantismo contemporaneo resta quello di un atteggiamento del poeta fondamentalmente reazionario e incapace di misurarsi con i tempi. ${ }^{71}$ Viene in mente, di fronte a questa diagnosi ultimativa, la divertita ironia con cui Gadda commentava il giudizio di un Manzoni reazionario e conservatore espresso da Moravia in un suo celebre intervento critico, ${ }^{72}$ in cui l'autore dei Promessi Sposi veniva accusato di avere rappresentato un seicento lombardo borromeiano e cattolico: «cattolico era, lui non poteva farlo turco», dice Gadda. ${ }^{73}$ E così, parafrasando, il medioevo di Dante «cattolico era, lui non poteva

${ }^{70}$ La perifrasi contenuta nei versi $142-143$ si riferisce allo scarto di dodici minuti fra la durata dell'anno secondo il calendario giuliano (365 giorni e 6 ore) e la durata dell'anno reale (365 giorni, 5 ore e 48 minuti). Ogni anno veniva dunque esclusa dal computo la centesima parte di un giorno («la centesma ch'è laggiù negletta»). Ora, affinché si verifichi un accumulo di tempo tale da spostare gennaio in primavera sarebbero necessari novanta secoli: la profezia di Beatrice va dunque interpretata come l'affermazione, in figura appunto di litote, che l'evento profetizzato si verificherà fra breve.

${ }^{71}$ Un caso esemplare di questo giudizio lo troviamo nella biografia di Dante di Marco Santagata: «Ebbene, l'intellettuale che mostra di avere una così acuta percezione della storicità dei fenomeni culturali, quando volge lo sguardo alle dinamiche sociali, economiche e politiche della sua epoca vorrebbe bloccare il corso della storia, anzi, riportare indietro le lancette dell'orologio. Rifiuta in blocco gli assetti produttivi basati sulla manifattura, il commercio e la finanza, il rimescolamento del tessuto sociale dei Comuni da essi prodotto (la «cittadinanza ch'è or mista»), le nuove forme signorili di governo (che lui chiama «tirannidi»), il deperimento delle giurisdizioni feudali, la centralità della finanza nei rapporti tra Stati e signorie. Dante considera il dinamismo sociale degenerazione dei costumi e perversione dei valori; la perdita di ruolo e di potere degli antichi ceti dominanti, caduta dei pilastri dell'ordine comunitario; la concorrenza aspra tra le città e l'affermarsi di istituzioni signorili, disordine esiziale per la pacifica convivenza della cristianità. È convinto che la salvezza verrà solo ritornando indietro alla serena e domestica Firenze premercantile, all'epoca in cui la cristianità poggiava sull'equilibrio tra i due «soli» (papato e impero), a un assetto sociale gerarchico e stabile imperniato sulla nobiltà feudale. Tornare indietro, bloccare il tempo. Ricostituire un mondo immobile, garantito da un disegno istituzionale immutabile, simile in questo all'eterna corte celeste del Paradiso», Santagata, Dante. Il romanzo della sua vita, op. cit., p. 14.

${ }^{72}$ Moravia, A., Alessandro Manzoni e l'ipotesi di un realismo cattolico, in L'uomo come fine e altri saggi, Milano, Bompiani, 1963, pp. 167-205.

${ }^{73}$ Gadda, C.E., Manzoni diviso in tre dal bisturi di Moravia, in Il tempo e le opere, Milano, Adelphi, 
farlo socialdemocratico». Tutt'al più poteva reagire, come fece, allo strapotere della lupa e del «maladetto fiore»: e non sembra davvero poco.

Al cuore dell'equivoco ci sono con tutta evidenza i canti di Cacciaguida: è quello il cantiere in cui viene reperita la gran parte dei materiali con cui si è costruito il monumento del «Dante reazionario», dalla descrizione della Firenze «sobria e pudica» e dei suoi leggendari protagonisti(Par. XV, 97-135) alla deprecazione della cittadinanza «mista» e inquinata dall'inurbamento (Par. XVI, 49-57), dalla condanna della «confusion de le persone» (Par. XVI, 67-68) all'elenco celebrativo delle antiche famiglie fiorentine (Par. XVI, 88-154).

Certo, di fronte a questa successione di exempla sembra davvero difficile evitare l'impressione di una ottusa laudatio temporis acti e di una ostinata cecità di Dante rispetto alla gigantesca crisi che era in atto sotto i suoi occhi; e però, secondo il buon costume storico-filologico, per una corretta valutazione bisognerebbe sottrarsi alle categorie del presente e riportarsi alle coordinate culturali e morali su cui si orienta il pensiero dantesco. Vedremmo allora che non siamo affatto di fronte a un caso di cieco conservatorismo, ma al progetto di una radicale alternativa di sistema che si fonda sulla proiezione, in un futuro avvertito come prossimo, dei valori di sobrietà, giustizia e libertà recuperati dalla tradizione della res publica romana e inseriti, attraverso l'etica del dono e il pauperismo evangelico-francescano, nel quadro della charitas cristiana. Il mito dell'impero non è altro che la sintesi operativa di questo progetto, ben lontano quindi dal rappresentare una mediocre utopia reazionaria ma tutto centrato sulla grandiosa concezione di un mondo rigenerato a partire dai rapporti sociali e politici che lo determinano. Un ben strano reazionario, insomma, questo Dante che combatte lo stato di cose presente non fantasticando di un impossibile (e grottesco) ritorno al passato, ma evocando un futuro di prodigiosa diversità, proiettato in una grandiosa dimensione di utopia e saldamente attestato sul versante teologico e profetico, terreno inospite per quella nostalgia del buon tempo antico che è al fondo di ogni sogno reazionario.

Ecco dunque che i canti di Cacciaguida non esprimono chiusura municipalistica e gretto conservatorismo, ma rivelano un piglio profetico di matrice scritturale che acquista potenza ispirandosi a fonti classiche bene individuabili, in primo luogo il Sallustio del De Catilinae coniuratione, come dimostra in modo inoppugnabile Francesca Fontanella in un suo pregevole studio. ${ }^{74}$ Sulla stessa linea si collocano Charles T. Davis,

\footnotetext{
1982, p. 34.

${ }^{74}$ «Il contrasto fra la città di un tempo 'in pace, sobria e pudica' con la città contemporanea al poeta dove regnano il lusso e la lussuria, suggerisce che Dante, nel tratteggiare questo contrasto, seguisse ancora gli autori classici che esaltavano la virtus dell'antica res publica romana contrapponendola al decadimento dei costumi e all'imperversare dei contrasti civili. La virtuosa Firenze delineata dal discorso di Cacciaguida, infatti, in cui è sempre presente sullo sfondo la successiva decadenza della città, è sì una riproposizione del mito dell'età dell'oro, ma in chiave risolutamente cittadina e fitta di reminiscenze romaneggianti. Così, per citare solo qualche passo
} 
che attribuisce analoghe funzioni di riferimento ideale a Virgilio e a Giovenale, ${ }^{75} \mathrm{e}$ Robert Hollander, che individua in Lucano la principale fonte del repubblicanesimo di Dante. ${ }^{76}$ Per quanto riguarda influenze e suggestioni sul tema del 'buon tempo antico' che Dante può avere ricevuto dai contemporanei, ancora Davis attribuisce grande importanza a una pagina delle Historie di Riccobaldo da Ferrara ${ }^{77}$ (o meglio, del

degli autori antichi che Dante poteva conoscere, ricordo che nella Congiura di Catilina, prima dell'excursus «archeologico», Sallustio parla dei corrupti mores della città, nella quale imperavano due vizi diversi, ma parimenti funesti, la luxuria e l'avaritia. Dopo la distruzione di Cartagine, infatti, 'prima aumentò la sete di denaro, quindi quella di potere e queste furono la causa di quasi tutti i mali. Infatti l'avaritia stravolse la fides, l'onestà e tutte le buone attitudini e insegnò al loro posto la superbia, la crudeltà, a trascurare gli dèi, a considerare tutto in vendita e comprabile'. Verrebbe da dire: «dantesca« questa insistenza di Sallustio sull'avarizia, non solo come sete di denaro ma anche di dominio, e pertanto causa di tutti i mali! E leggiamo poco più avanti: 'Dopo che le ricchezze iniziarono a essere notivo di onore e ad esse seguivano la gloria, il comando e il potere, la virtù iniziò a illanguidirsi, la povertà a essere ritenuta motivo di disonore, l'integrità iniziò a essere interpretata come un'ostentazione malevola. Quindi, dalle ricchezze si riversarono fra i giovani la luxuria e l'avaritia insieme alla superbia': ecco, in un diverso ordine, le tre fiere del primo canto dell'Inferno [...] E sempre Sallustio parla anche della smisurata grandezza di case e di ville 'costruite a misura di città' e della lussuria (lubido) che dilagava fra uomini e donne al posto della pudicitia», Fontanella, F., L'impero e la storia di Roma in Dante, Bologna, Il Mulino, 2016, pp. 323325.

${ }^{75}$ «Tuttavia, se Dante si oppose alla corrente storiografica del proprio comune, riecheggiò un topos consueto degli storici, degli oratori e dei poeti di Roma. Può aver ricordato il passo virgiliano del libro II delle Gerogiche, che condannava la decadenza moderna ed elogiava il tempo governato dall'aureus Saturnus, quando Roma, racchiusa in piccole mura, non si era ancora votata all'acquisto del potere e della ricchezza. Può aver pensato al quadro tracciato da Giovenale nelle Satire (tra le quali Dante sicuramente conosceva la VII, l'VIII e la IX) di una metropoli corrotta e licenziosa», Davis, C.T., Il buon tempo antico, in L'Italia di Dante, Bologna, Il Mulino, 1988, pp. 108-133, alla p. 128.

${ }^{76} \mathrm{Hollander}$, R. e Rossi, A., Il repubblicanesimo di Dante, in G.C. Alessio e R. Hollander (eds.), Studi americani su Dante, Milano, Franco Angeli, 1989, pp. 297-323. Lo studio è pregevole, anche se sviluppato con qualche forzatura e qualche incertezza ermeneutica, come quando, per esempio, il v. 106 di Par. XV (Non avea case di famiglia vòte) viene letto come un'allusione alle «abitazioni pompose» (p.317), con un evidente equivoco interpretativo.

${ }^{77}$ «Per hec tempora rudes erant in Italia ritus et mores. Nam viri infulas de squamis ferreis capite gestabant insutas biretis quas appellabant maiatas. In cena vir et uxor una manducabant paraside; usus incisorum ligneorum non erat in mensis. Unus vel duo scyphi erant ad potum in familia. Nocte cenantes mensas facibus illustrabant, facem tenente uno puerorum vel servo; candelarum enim de sepo vel de cera usus non erat. Viri clamidibus pelliceis sine operimento, vel laneis sine pellibus et infulis de pignolato utebantur, femine nubentes tunicis de pignolato vestiebantur. Viles tunc erant cultus virorum rt feminarum. Aurum et argentum rarum vel nullum erat in vestibus. Ciborum lautia nulla. Homines plebeii ter in septimana carnibus recentibus vescebantur; tunc prandio ederant holera vel legumina cum carnibus cocta, cenam autem ducebant ipsis carnibus reservatis. Non omnibus erat usus vini estate. Modica nummorum summa se locupletes tenebant. Parve tunc erant celle vinarie; horrea non ampla habebant, promptuariis contenti. Dote modica nubebant femine, quia earum cultus tunc erat parcissimus. Virgines ante nuptias tunica de pignolato que dicebatur sotanum et paludamento lineo quod xocham dicebant erant contente. Ornatus capitis non preciosus erat virginibus. Matrone vitis latis 
Compendium, visto che la seconda parte delle Historie in cui era contenuto il passo in questione è andata perduta). L'ipotesi è suggestiva, ma va detto che dopo lo studio di Mirko Tavoni ${ }^{78}$ che dimostra la dipendenza di Riccobaldo da Dante, e non viceversa, la tesi di Davis (comunque precedente al saggio di Tavoni) sembrerebbe perdere attendibilità. In ogni caso il passo di Riccobaldo rappresenta, quello sì, un caso esemplare di laudatio temporis acti senza nessuna valenza progettuale e nessuna prospettiva di cambiamento che non sia quella di tornare a un immaginario buon tempo antico: e ci sembra che, a commento del mondo ideale rimpianto da Riccobaldo, si prestino perfettamente le parole di un grande medievalista, Roberto Lopez, che così descriveva la società urbana prima della rivoluzione mercantile:

È il mondo della botteguccia, del piccolo artigianato, del commercio al minuto per il mercato ristretto del quartiere o della città. Qui, i regolamenti corporativi sono osservati in tutta la loro saggezza, ma anche in tutta la loro angustia. I maestri seguono le orme dei propri padri, , maestri del medesimo mestiere; i garzoni si sforzano di diventar maestri, senza altra ambizione che quella di conservare i buoni metodi tradizionali. In questo ambiente di tranquilla mediocrità, la disonestà stessa tende ad assumere forme modeste, quasi bonarie: pesi e misure contraffatte, merci avariate, $\mathrm{o}$, come nel caso di un fornaio inglese che fu colto in flagrante, un'apertura nel banco attraverso la quale far scivolare furtivamente, nelle mani di un garzone accovacciato lì sotto, una parte della pasta che i clienti portano a far cuocere. Le distrazioni consistono in una sosta domenicale all'osteria, una partita a dadi per i più avventurosi, qualche processione, qualche festa religiosa. Nell'insieme, una vita monotona e senza scosse. ${ }^{79}$

Certo, non è a questo mondo che intende tornare Dante, né la sua palingenesi si fonda su un'operazione di ingegneria istituzionale, come dimostra l'apostrofe a Firenze che chiude il sesto canto del Purgatorio (145-151): «Quante volte, del tempo che rimembre, / legge, moneta, officio e costume / hai tu mutato, e rinovate membre! / E se ben ti ricordi e vedi lume, / vedrai te somigliante a quella inferma / che non può

tempora et genas cum mento vittabant. Virorum erat gloria esse in armis et equis locupletes. Nobilium gloria erat turres excelsas habere. Multa nunc inhonesta superindicta sunt rebus priscis, verum plurima ad perniciem animarum. Mutata est parsimonia in lautiam. Materia et artificio exquisito nimioque ornata vestimenta cernuntur. Illic argentum aurum et margarite mira fabricata. Frigia latissima, fulcimenta vestium serica vel varia pellibus exoticis ac preciosis. Irritamenta gule non desunt. Vina peregrina bibuntur, fere omnes potores in publico. Obsonia sumptuosa, eorum magistri coquinarii habuntur in magno precio. Omnia ad gule irritamenta et ambitionis queruntur. Ut his suppeditari possit, avaricia militat. Hinc usure, fraudes, rapine, expilationes, prede, contentiones, in re publica vectigalia illicita, innocentem oppressiones, exterminia civium, relegationes locupletum. Venter noster deus est noster. Pompis quibus renunciavimus in batismo insistimus, facti adeo transfuge ad zabulum. Et nisi clericorum disciplina nos iugiter castis exemplis instrueret, ambitioni et deliciis nostris modum non esset», Ricobaldi Ferrariensis, Compendium Romanae Historiae, Roma, Istituto Storico Italiano per il Medioevo, 1984, II, pp. 723-724.

${ }^{78}$ Tavoni, Riccobaldo da Ferrara fonte di Dante?, in Qualche idea su Dante, op. cit., pp. 260-264.

${ }^{79}$ Lopez, R.S., La nascita dell'Europa, Torino, Einaudi, 1966, pp. 315-316. 
trovar posa in su le piume, / ma con dar volta suo dolore scherma». Non è questione, dunque, né di tornare al passato né di mutare «legge, moneta, officio e costume», ma di rivoluzionare, attraverso l'ordinamento imperiale, gli assetti sociali, economici e politici della cristianità, partendo da un principio che doveva suonare scandaloso alle orecchie dei fiorentini fra XIII e XIV secolo: che la ricchezza, cioè, è un male in sé e che non è possibile né pace né giustizia là dove essa costituisce il valore fondante di una comunità e, sfuggendo a ogni controllo, si trasforma fatalmente in avarizia che alimenta la cupidigia. Principio scandaloso, se ricordiamo l'esergo con cui si aprivano i libri della ragione della Compagnia fiorentina dei Peruzzi: «In nome di guadagno e salvamento in mare e in terra e accrescimento di avere e di persone con salute de l'anima e de' corpi nostri». Ebbene, per la nuova economia politica proposta da Dante, «guadagno» e «accrescimento di avere» non sono i valori in nome dei quali vale la pena vivere, ma, al contrario, le fonti della cupidigia che avvelena il mondo e, di conseguenza, di quella infelicità che sembra pesare come una condanna ineluttabile sul vivere umano. Ecco come, nel quarto trattato del Convivio, Dante individua e descrive con esemplare chiarezza le radici dell'infelicità umana:

Onde, con ciò sia cosa che l'animo umano in terminata possessione di terra non si queti, ma sempre desideri gloria d'acquistare, sì come per esperienza vedemo, discordie e guerre conviene surgere intra regno e regno, le quali sono tribulazioni de le cittadi, e per le cittadi de le vicinanze, e per le vicinanze de le case, e per le case de l'uomo; e così s'impedisce la felicitade. ${ }^{80}$

Come sarà possibile, allora, ritrovare la strada per questa «felicitade» impedita dalla bramosia del possesso? La risposta è data subito dopo:

Il perché, a queste guerre e le loro cagioni torre via, conviene di necessitade tutta la terra, e quanto a l'umana generazione a possedere è dato, essere Monarchia, cioè uno solo principato, e uno principe avere; lo quale, tutto possedendo e più desiderare non possendo, li regi trgna contenti ne li termini de li regni, sì che pace intra loro sia, ne la quale si posino le cittadi, e in questa posa le vicinanze s'amino, in questo amore le case prendano ogni loro bisogno, lo qual preso, l'uomo viva felicemente; che è quello per che esso è nato. ${ }^{81}$

L'impero, dunque, non come riproposizione mitica e mistica di una leggenda ormai fuori dalla storia, ma come prassi organizzativa in grado di rispondere vittoriosamente al drammatico incombere della lupa che condanna gli uomini all'infelicità. Colpisce l'insistenza sul tema della felicità umana (beatitudo huius vitae) non come obiettivo da raggiungere nella patria celeste, ma possibile qui e ora, su questa terra, come si teorizza esplicitamente nel De Monarchia ${ }^{82}$ (ma il tema della felicità terrena, associato all'elogio

${ }^{80}$ Conv. IV, 4

${ }^{81} \mathrm{Ibid}$.

82 «Duos igitur fines providentia illa inenarrabilis homini proposuit intendendos: beatitudinem scilicet huius vitae, que in operatione proprie virtutis consistit et per terrestrem 
dell'austerità, è presente anche in san Tommaso $\left.{ }^{83}\right)$. Ė un punto essenziale che contraddice ogni interpretazione di Dante come assertore di quell'ideale ascetico, pauperistico e cupamente penitenziale che pare a molti l'unica alternativa all'economia mercantile (oggi si direbbe «liberista»): al contrario, nel mondo liberato dai vincoli della cupidigia e dal pensiero unico utilitarista ci sarà posto per la gioia di vivere e per quella felicità a cui l'uomo è destinato («No, il comunismo non oscurerà la bellezza e la grazia» dirà Antonio Gramsci sei secoli dopo, rispondendo a obiezioni analoghe). L'esempio del mercante, addotto sempre nel quarto trattato del Convivio, non lascia dubbi sull'effetto di felicità provocato dall'abbandono della cupidigia:

Quanta paura è quella di colui che appo sé sente ricchezza, in camminando, in soggiornando, non pur vegliando ma dormendo, non pur di perdere l'avere ma la persona per l'avere! Ben lo sanno li miseri mercatanti che per lo mondo vanno, che le foglie che 'l vento fa menare, li fa tremare, quando seco ricchezze portano; e quando sanza esse sono, pieni di sicurtade, cantando e sollazzando fanno loro cammino più brieve. ${ }^{84}$

Davvero «conviviale» (nel senso attribuito al termine da Ivan Illich ${ }^{85}$ ) questa immagine del mercante in cammino per le vie del mondo "cantando e sollazzando», liberato dal peso oppressivo della cupidigia e delle «divizie»! E certo rivive qui, a distanza di molti anni, quell'ideale di una vita libera e lieta che Dante aveva coltivato ai tempi della giovinezza, quando aveva sognato di correre le vie del mare su un vascello incantato dove amore e amicizia fossero all'origine della comune felicità. Ma quello che negli anni stilnovistici era un vagheggiamento poetico o, al massimo, un progetto di vita destinato a una ristretta comunità, si è fatto col tempo un programma concreto di trasformazione della società. Il punto di snodo che segna il momento di passaggio da una dimensione all'altra è la conquista della coscienza che la costruzione della «società conviviale» è possibile solo a partire da una condizione: la distruzione del meccanismo perverso che fa della ricerca dell'utile il primo e unico valore su cui orientare la vita, e questo risultato sarà possibile solo cancellando dall'animo umano l'ossessione del possesso e la conseguente cupidigia: «Ubi ergo non est quod possit optari, inpossibile

paradisum figuratur; et beatitudinem vite ecterne, que consistit in fruitione divini aspectus ad quam propria visrtus ascendere non potest, nisi lumine divino adiuta, que per paradisum celestem intelligi datur. Ad has quidem beatitudines, velut ad diversas conclusiones, per diversa media venire oportet. Nam ad primam per phylosophica documenta venimus, dummodo illa sequamur secundum virtutes morales et intellectuales operando; ad secundam vero per documenta spiritualia que humanam rationem trascendunt, dummodo illa sequamur secundum virtutes theologicas operando, fidem spem scilicet et karitatem», Mon. III, 15.

${ }^{83}$ «Austeritas secundum quod est virtus non excludit omnes delectationes, sed superfluas et inordinatas: unde videtur pertinere ad affabilitatem, quam Philosophus, lib 4 Ethic. Cap.VI, «amicitiam» nominat, vel ad eutrapeliam, sive jocunditatem», Tommaso d'Aquino, Summa Theologiae II, 2, quest. CLXVIII, art. 4.

${ }^{84}$ Conv. IV, 13.

${ }^{85}$ Illich, I., La convivialità, Milano, Mondadori, 1974. 
est ibi cupiditatem esse: destructis enim obiectis, passiones esse non possunt». ${ }^{86}$ Questa situazione ideale in cui, tramite l'estinzione del desiderio e la scomparsa della cupidigia, può instaurarsi il regno della felicità e della giustizia si determina appunto con l'instaurazione della Monarchia universale:

Sed Monarchia non habet quod possit optare: sua nanque iurisdictio terminatur Occeano solum: quod non contingit principibus aliis, quorum principatus ad alios terminantur, ut puta regis Castellae ad illum qui regis Aragonum. Ex quo sequitur quod Monarcha sincerissimus inter mortales iustitie possit esse subiectum. Preterea, quemadmodum cupiditas habitualem iustitiam quodammodo, quantumcunque pauca, obnubilat, sic karitas seu recta dilectio illam acuit atque dilucidat. Cui ergo maxime recta dilectio inesse potest, potissimum locum in illo potest habere iustitia: hiusmodi est Monarchia: ergo, eo existente, iustitia potissima est vel esse potest. ${ }^{87}$

Gli strumenti con cui attuare questa precondizione, cioè far sì che gli uomini accettino di riconoscere il Monarca, sono disponibili da subito: in primo luogo l'affermarsi della recta dilectio, cioè del dominio della charitas, da cui discendono l'orientamento legislativo verso il bene comune, e contestualmente l'applicazione dell'etica del dono come prassi economica:

Quod autem recta dilectio faciat quod dictum est, hic haberi potest: cupiditas nanque, perseitate hominum spreta, querit alia; karitas vero, spretis aliis omnibus, querit Deum et hominem, et per consequens bonum hominis. ${ }^{88}$

È evidente qui la presenza della riflessione agostiniana sui due amori che si contendono l'anima umana, l'«amor sui» e l'«amor Dei» («Fecerunt itaque civitates duas amores duo, terrenam scilicet amor sui usque ad contemptum Dei, coelestem vero amor Dei usque ad contemptum sui») ${ }^{89}$ ed è del pari evidente come l' « amor Dei» possa concretarsi solo attraverso la sostituzione dell’etica dell'utilitarismo con quella della charitas e con la conseguente scomparsa dell' «amor sui» e della cupidigia

Insomma, appare chiaro come nell'economia politica dantesca il ruolo del profitto e dell'utile individuale venga ridimensionato passando da valore centrale dell'esistenza a componente secondaria, ancorché necessaria al vivere associato: mai Dante, infatti, ha pensato all'abolizione tout court di ogni forma di profitto, ma a una sua nuova dislocazione nella scala dei valori che subordini le «divizie» alla charitas e al bene comune, pur riconoscendone la necessità nella misura in cui esse «ad alcuno necessario servizio sono ordinate». ${ }^{90}$ Nessun integralismo, quindi, ma una ridefinizione dei rapporti sociali che liberi l'umanità dalla schiavitù del denaro. In questo senso vanno letti i canti di Cacciaguida, troppe volte interpretati come esempio di conservatorismo

\footnotetext{
${ }^{86}$ Mon., I, 11.

${ }^{87}$ Ibidem.

${ }^{88}$ Ibidem.

${ }^{89}$ Agostino, De Civitate Dei XIV, 28.

${ }^{90}$ Conv. IV, 13.
} 
reazionario, a partire dalla famigerata «confusion de le persone» che non è affatto un invito a chiudersi in un angusto orizzonte municipale ignorando ottusamente l'evoluzione della storia, ma la denuncia della «dismisura» con cui Firenze, accecata dalla bramosia di potenza e dalla sete di guadagno, si è lanciata in una dissennata politica di conquista che ha fatalmente portato alla degenerazione e al crollo dei rapporti sociali in direzione dell'egoismo utilitarista. Si tratta di una diagnosi di ascendenza tomistico-aristotelica, ${ }^{91}$ come dimostra in modo convincente Elisa Brilli in un suo documentatissimo studio, ${ }^{92} \mathrm{e}$ per niente affatto di una manifestazione di cecità politica a sfondo reazionario.

Possiamo a questo punto legittimamente attribuire a Dante il progetto di un nuovo modello di società alla cui base vi sia il consapevole ripudio dell'utilitarismo, cioè di quell'atteggiamento che induce a condizionare le proprie scelte di vita al vantaggio e al profitto individuale che di volta in volta è possibile trarne? La forma interrogativa in cui si propone questa ipotesi nasce dal fatto che l'interpretazione in chiave antiutilitarista dell'economia politica dantesca potrebbe sembrare una forzatura: in effetti, per avere una teoria compiuta del pensiero utilitarista dovremo attendere il XVIII secolo, quando Jeremy Bentham, nel suo saggio An Introduction to the Principles of Morals and Legislation (1789), elaborerà la dottrina per cui gli uomini sono guidati dalla logica egoista del calcolo dei piaceri e dei dolori, ed è bene che sia così perché «non esiste altro fondamento possibile delle norme etiche se non la legge della felicità degli individui o della collettività degli individui»»3. La formula abitualmente usata per esprimere l'essenza del pensiero di Bentham (formula che peraltro il filosofo inglese

${ }^{91}$ «Dignior enim est civitas si abundantiam rerum habeat ex territorio proprio, quam si per mercatores abundet; cum hoc etiam videatur esse securius, quia propter bellorum eventus et diversa viarum discrimina, de facili potest impediri victualium deportatio, et sic civitas per defectum victualium opprimetur. Est etiam hoc utilius ad conversationem civilem. Nam civitas quae ad sui sustentationem mercationum multitudine indiget, necesse est ut continuum extraneorum convictum patiatur. Extraneorum autem conversatio corrumpit plurimum civium mores, secundum Aristotelis doctrinam in sua Politica, quia necesse est evenire ut homines extranei aliis legibus et consuetudinibus enutriti, in multis aliter agant quam sint civium mores, et sic, dum cives exemplo ad agenda similia provocantur, civilis conversatio perturbatur. Rursus: si cives ipsi mercationibus fuerint dediti, pandetur pluribus vitiis aditus.Nam, cum negotiatorum studium maxime ad lucrum tendat, per negotiationis usum cupiditas in cordibus civium traducitur, ex quo convenit, ut in civitate omnia fiant venalia, et fide subtracta, locus fraudibus aperitur, publicoque bono contempto, proprio commodo quisque deserviet, deficietque virtutis studium, dum honor virtutis praemium omnibus deferetur: unde necesse erit in tali civitate civilem conversationem corrumpi. Denique civitas illa solet esse magis pacifica, cuius populus rarius congregatur, minusque intra urbis moenia residet. Ex frequenti enim hominum concursu datur occasio litibus et seditionibus materia ministratur. Unde secundum Aristotelis doctrinam, utilius est quidem quod populus extra civitates exerceatur, quam quod intra civitatis moenia iugiter commoretur», Tommaso d'Aquino, De regno ad regem Cypri, II, 3.

${ }_{92}$ Brilli, E., Firenze e il profeta. Dante fra teologia e politica, Roma, Carocci, 2012. Cfr. in particolare le pp. 128-139.

${ }^{93}$ Caillé, A., Critica della ragione utilitaria, Torino, Bollati Boringhieri, 2005, p. 13. 
aveva mutuato da Cesare Beccaria) è «la massima felicità per il maggior numero», sintesi di una logica utilitarista che peraltro si era affermata già a partire dal basso medioevo. Certo, al tempo di Dante l'utilitarismo non era ancora quello che poi è diventato dopo Bentham, cioè il fondamento normativo comune a tutto il pensiero moderno, ma la sua presenza, ancorché in forme ancora allo stato nascente, è già potente soprattutto in realtà come quella fiorentina dominata dalle logiche mercantili e finanziarie fin dagli inizi del XIII secolo. ${ }^{94}$ Quanto all'antiutilitarismo, dobbiamo spostarci in anni ancora più recenti per coglierne una presenza teoricamente organizzata: si pensi alle indagini antropologiche di Marcel Mauss (1872-1950) in cui le pretese universalistiche della moderna economia politica di ispirazione utilitarista vengono smascherate e ridefinite come puri prodotti sovrastrutturali privi di ogni oggettività, a cui occorre contrapporre il ritorno a un'etica del dono e del disinteresse. ${ }^{95}$ E tuttavia, anche in questo caso non è pretestuoso individuare nella polemica medievale contro il prestito a interesse e la mercatura, nella condanna della cupidigia e nelle correnti pauperistiche che affondano le radici fino nell'antichità, una evidente affinità con la critica moderna all'egoismo consumistico e al mito della crescita senza limiti (la «dismisura» dantesca). Non sembra dunque improprio cogliere in Dante un vivissimo riflesso di questa polemica, un riflesso certo privo di consapevolezza teorica, ma vissuto drammaticamente come l'incombere minaccioso di forze ignote e proprio per questo ancora più sinistre (si pensi a un testo come la canzone Doglia mi reca).

Diciamo però con la massima chiarezza che è lontanissimo dalle nostre intenzioni fare di Dante un precursore dell'antiutilitarismo contemporaneo: riguardo al «precursorismo», dilagante in anni non lontani e oggi per fortuna meno invadente, la

${ }^{94}$ «Fernand Braudel, per esempio, ritiene che sin dal secolo XIII e forse addirittura dall'XI, l'economia europea sia assoggettata alla legge del mercato. Questa sarebbe già largamente all'opera in India, in Cina e nell'Impero ottomano», ibid., p. 66.

${ }_{95}$ «Noi torniamo, ed è necessario tornarvi, a una pratica di «spesa nobile». Occorre che i ricchi, come nei paesi anglosassoni, come in tante altre società contemporanee, selvagge e altamente civilizzate, tornino -liberamente e anche forzatamente- a considerarsi come una specie di tesorieri dei propri concittadini. Le civiltà antiche -dalle quali discendono le nostre- avevano, le une il giubileo, le altre le liturgie, coregie e trierarchie, le sussitie (pasti in comune), le spese obbligatorie dell'edile e dei personaggi consolari. Sarà necessario risalire a leggi di questo tipo. Occorre, inoltre, una maggior cura dell'individuo, della sua vita, della sua salute, della sua educazione -cosa utile, del resto- della sua famiglia e dell'avvenire di quest'ultima. Occorre più buona fede, più sensibilità, più generosità nei contratti di lavoro, nelle locazioni di immobili, nella vendita di generi necessari. E bisognerà trovare il mezzo per limitare i frutti della speculazione $\mathrm{e}$ dell'usura. [...] Si può e si deve, perciò, tornare a qualcosa di arcaico; si ritroveranno così motivi di vita e di azione ancora familiari a società e classi numerose: la gioia di dare in pubblico; il piacere del mecenatismo; quello dell'ospitalità e delle faste private e pubbliche. La sicurezza sociale, la sollecitudine mutualistica, cooperativa, del gruppo professionale di tutte quelle istituzioni che il diritto inglese fregia del nome di «Friendly Societies»; valgono di più della semplice sicurezza personale che il nobile garantiva al suo fittavolo, di più della vita meschina offerta dal salario giornaliero del datore di lavoro, e anche di più del risparmio capitalistico fondato su un credito mutevole.», Mauss, Saggio sul dono, op. cit., pp. 273-274. 
nostra opinione coincide con quella espressa a suo tempo con la consueta verve polemica da Luigi Russo che lo considerava una manifestazione di pochezza critica e di debolezza morale. ${ }^{96} \mathrm{E}$ quindi non cadremo nel pressapochismo di vedere in Dante un precursore di Marcel Mauss, di Ivan Illich o di Serge Latouche.

Detto questo, è fuor di dubbio che nell'opera dantesca siano presenti, senza «precorrere» alcunché ma semplicemente come risposta a una condizione di disagio morale e come risultato di un'analisi storica condotta «sul campo», forti elementi di antiutilitarismo che alla fine risultano fondativi rispetto al suo progetto palingenetico. E non sorprende che questo atteggiamento, entrando in conflitto con il mainstream utilitarista della sensibilità contemporanea, sia stato scambiato per una manifestazione di conservatorismo reazionario. Casomai, potrà stupire il fatto che una questione oggi al centro del dibattito come l'obiezione antiutilitarista sia anche al centro della riflessione dantesca. Ma qui converrà richiamarsi alla splendida considerazione di Gianfranco Contini, quando osserva che «limpressione genuina del postero, incontrandosi in Dante, non è d'imbattersi in un tenace e benconservato sopravvissuto, ma di raggiungere qualcuno arrivato prima di lui». ${ }^{97}$ Dunque, non è Dante il precursore, ma probabilmente siamo noi i ritardatari.

Marco Romanelli cosmar46@yahoo.it

Fecha de recepción: 25/10/2019

Fecha de aceptación: 04/02/2020

\footnotetext{
${ }^{96}$ «Diremo, in genere, che questa tendenza a trovar dei precursori dovunque è, innanzi tutto, segno di povertà storica e morale. Quando un movimento non è ricco di pensiero o di tradizione o ha esaurito il suo motivo creatore, allora saltan fuori i celebratori delle forze precorritrici, i ritrovatori di blasoni, gli scopritori degli antesignani, i letteratucoli testimonianti sui sepolcri illustri... In fondo, questa giacobina contrazione del passato nel nostro presente è un disconoscimento del valore religioso degli acquisti della civiltà umana, i quali sono lentissimi, e mai l'uno è ripetizione dell'altro; sono un disconoscimento delle nostre stesse virtù attuali. Il sentimento profondo è sempre senso della distinzione storica, e la lenta formazione degli ideali, attraverso i secoli, è riprova della loro verità e della loro concretezza e necessità, e il voler contrarre l'esperienza nostra con quella dei nostri antenati, e il voler fare di successive esperienze una sola cosa, un solo idolo, sta a testimoniare superficialità e incoscienza della fatica e importanza dei nostri acquisti e della nostra originalità presente.», Russo, L., Machiavelli, Bari, Laterza, 1969, p. 186.

${ }^{97}$ Contini, G., Un'interpretazione di Dante, in Un'idea di Dante, Torino, Einaudi, 1976, pp. 69-111, alla p. 111.
} 